\title{
Two-dimensional mass spectrometry: new perspectives for tandem mass spectrometry
}

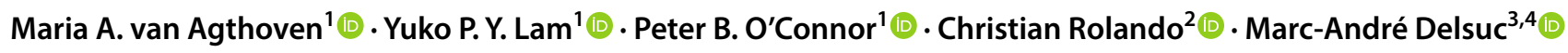

Received: 24 August 2018 / Revised: 24 January 2019 / Accepted: 6 February 2019 / Published online: 13 March 2019

(c) The Author(s) 2019

\begin{abstract}
Fourier transform ion cyclotron resonance mass analysers (FT-ICR MS) can offer the highest resolutions and mass accuracies in mass spectrometry. Mass spectra acquired in an FT-ICR MS can yield accurate elemental compositions of all compounds in a complex sample. Fragmentation caused by ion-neutral, ion-electron, or ion-photon interactions leads to more detailed structural information on compounds. The most often used method to correlate compounds and their fragment ions is to isolate the precursor ions from the sample before fragmentation. Two-dimensional mass spectrometry (2D MS) offers a method to correlate precursor and fragment ions without requiring precursor isolation. 2D MS therefore enables easy access to the fragmentation patterns of all compounds from complex samples. In this article, the principles of FT-ICR MS are reviewed and the 2D MS experiment is explained. Data processing for 2D MS is detailed, and the interpretation of 2D mass spectra is described.
\end{abstract}

Keywords Mass spectrometry · Two dimensional · Fourier transform · Fourier transform ion cyclotron resonance mass spectrometry · Tandem mass spectrometry

\section{Introduction}

In mass spectrometry, samples are ionized, separated according to mass-to-charge $(\mathrm{m} / \mathrm{z})$ ratio by modifying their trajectories and their velocities in an electric or magnetic field, and detected (de Hoffmann and Stroobant 2007). The measurement of their $\mathrm{m} / \mathrm{z}$ ratio gives information about the elemental composition of the compounds in the samples: the more accurate the $\mathrm{m} / \mathrm{z}$ ratio measurement, the more certainty there is about the elemental composition of the compounds. To quantify the confidence of the data interpretation, the resolution and the resolving power of individual peaks or

Marc-André Delsuc

madelsuc@unistra.fr

1 Department of Chemistry, University of Warwick, Gibbet Hill Road, Coventry CV47AL, UK

2 MSAP USR 3290, Université Lille, Sciences et Technologies, 59655 Villeneuve d'Ascq Cedex, France

3 Institut de Génétique, Biologie Moléculaire et Cellulaire, INSERM, U596, CNRS, UMR7104, Université de Strasbourg, 1 rue Laurent Fries, 67404 Illkirch-Graffenstaden, France

4 CASC4DE, 20 avenue du Neuhof, 67100 Strasbourg, France mass spectra are measured. In the IUPAC definition, the resolving power of a peak in a mass spectrum refers to the smallest mass difference between two peaks that can be differentiated. The resolution measures the ratio between the mass measurement and the resolving power. The accuracy of a mass assignment for a peak refers to the difference between the experimental and theoretical $\mathrm{m} / \mathrm{z}$ ratios divided by the theoretical $\mathrm{m} / \mathrm{z}$ ratio (Marshall et al. 1998).

In addition to measuring the $\mathrm{m} / \mathrm{z}$ ratio of compounds, mass spectrometers can also be used to fragment them to elucidate their chemical structure (tandem mass spectrometry) (Biemann 2015). Current fragmentation methods focus mainly on interactions with neutral particles, photons, and electrons. The ions from the sample are called precursors and the ions that are products of the fragmentation are called fragments. One important problem in tandem mass spectrometry of complex samples is how to correlate precursors and fragments to elucidate precursor structures accurately. The most common way to correlate precursors and fragments is to isolate a single $\mathrm{m} / \mathrm{z}$, ratio before fragmenting it (MS/MS) (Biemann 2002). MS/MS can be coupled with separation techniques to reduce the instantaneous complexity of the sample and to reduce ionization competition and Coulombic repulsion between ions (Peng et al. 2003; Ruger 
et al. 2017; Wootton et al. 2017). Because a choice is made for each MS/MS spectrum on which $\mathrm{m} / \mathrm{z}$ ratio is isolated and fragmented (a decision that is usually made algorithmically by the computer running the instrument: the acquisition switches between MS and MS/MS. In MS acquisition, the peaks with the highest intensity are identified and chosen to be isolated and fragmented. A mass exclusion list is generated and updated at every MS scan, so that a given ion species is not isolated and fragmented twice during the LC-MS/ MS run), MS/MS and MS/MS coupled with separation is called a data-dependent acquisition (or DDA) technique (Decaestecker et al. 2000). DDA is a very established technique that is well adapted to targeted analysis, in which the compounds of interest are determined before the analysis, for example in the case of pesticide analysis determined by regulations (Chapman et al. 2014).

However, for complex samples with multiple co-eluting species requiring analysis, data-independent acquisition (DIA) methods have been developed. One such method is precursor acquisition independent from ion count (PAcIFIC): ions go through a quadrupole in which a predetermined mass range is isolated, the isolated ions are then fragmented and analysed, and the mass range that is isolated is regularly incremented after each scan (Chapman et al. 2014; Panchaud et al. 2009). A similar technique has been commercialized as sequential windowed acquisition of all theoretical fragment ion mass spectra (SWATH-MS) by Sciex (Gillet et al. 2012). Another DIA technique, called $\mathrm{MS}^{\mathrm{E}}$, developed and commercialized by Waters, takes advantage of the fast duty cycle of time-of-flight analysers (1 ms) compared to the length of a chromatographic peak (10 s) by acquiring mass spectra with low and high fragmentation energy to correlate precursor and fragments (Cramer et al. 2016; Plumb et al. 2006).

A major issue of PAcIFIC/SWATH-MS is its reliance on ion isolation, which leads to experiment times and sample consumption that increase when the quadrupole isolation window (PAcIFIC) or the step size (SWATH) is decreased. Furthermore, because the width of the isolation windows is typically over $2 \mathrm{Da}$, separation between overlapping isotopic distributions with these methods is very difficult. In this paper, the progress of an alternative DIA method, twodimensional mass spectrometry (2D MS), is reviewed (van Agthoven et al. 2013). First, the general theory of the mass analyser on which 2D MS is developed, Fourier transform ion cyclotron resonance mass spectrometry (FT-ICR MS), is presented. Second, the principles of the 2D MS experiment and data processing are explained. Third, the interpretation of a 2D mass spectrum and the applications of 2D MS are described.

\section{Fourier transform ion cyclotron resonance mass spectrometry and tandem mass spectrometry}

Figure 1a shows a photograph of an FT-ICR mass spectrometer. The ion source is external to the mass analyser

(a) FT-ICR Mass Spectrometer with $12 \mathrm{~T}$

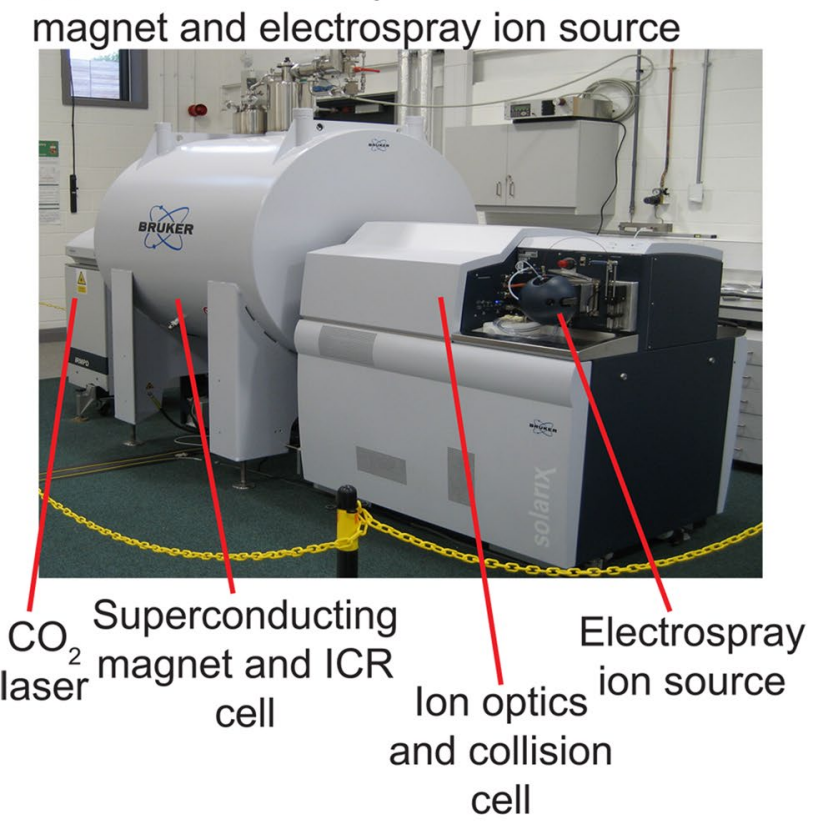

(b) Infinity ICR cell

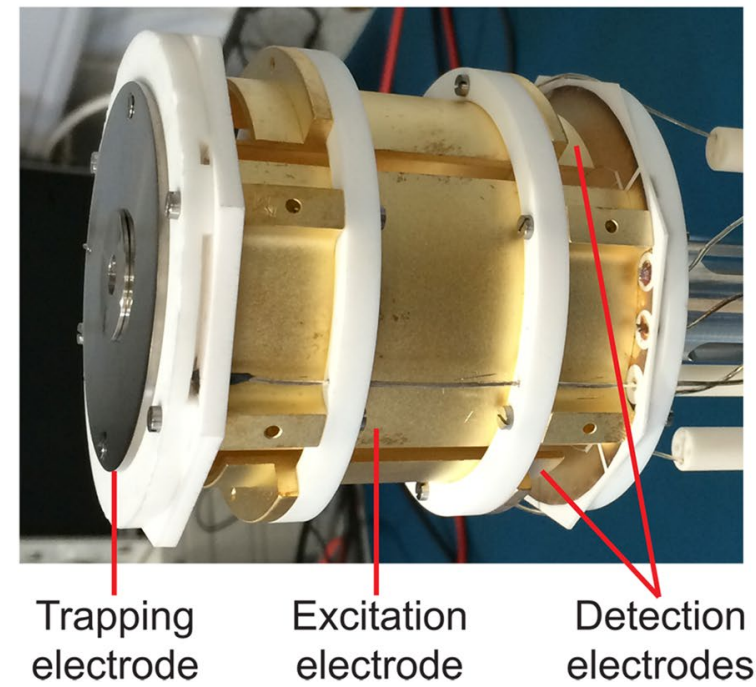

Fig. 1 a Photograph of a 12 T FT-ICR mass spectrometer with an electrospray ion source. Samples are ionized in the electrospray source and travel to the ICR cell in the centre of the superconducting magnet. The $\mathrm{CO}_{2}$ laser is used for fragmentation in the ICR cell. b Photograph of an Infinity ICR cell. The trapping electrodes are used to trap the ions axially. The excitation electrodes excite the ions to high radii and the detection electrodes detect mirror current generated by their motion 
and ions are transferred from the ion source to the mass analyser through ion optics. The mass analyser is an ion cyclotron resonance (ICR) cell situated in the centre of a superconducting magnet. A photograph of an ICR cell is shown in Fig. $1 b$.

FT-ICR mass spectrometry uses the cyclotron motion of ions inside a magnetic field. A homogeneous magnetic field induces a circular motion in ions with a frequency that can be expressed as:

$\omega_{\mathrm{c}}=\frac{q B}{m}$

in which $\omega_{\mathrm{c}}$ is the cyclotron frequency, $B$ the magnetic field, $q$ the charge of the ion and $m$ its mass. FT-ICR MS works by measuring the frequencies of ion motions in a homogeneous magnetic field and converting them into $\mathrm{m} / \mathrm{z}$ ratios (Amster 1996; Marshall et al. 1998). While Eq. 1 gives a good approximation (to 1 part in $10^{4}$ typically) of the frequency, the measured frequency will also be slightly shifted by the trapping electric fields, the pressure, and the Coulombic repulsion electric fields of the other ions that are simultaneously trapped. The resulting 'corrected' frequency is called the reduced cyclotron frequency and is fully considered in the true calibration equations (Zhang et al. 2005).

Ions are trapped inside an ICR cell made up of trapping electrodes, excitation electrodes, and detection electrodes (Vartanian et al. 1995), as shown in Fig. 1b. Figure 2 shows the process of acquiring and processing a mass spectrum inside an FT-ICR MS. In the simplest configuration, there are two electrodes for each function. Ions are trapped radially by the magnetic field and axially by the trapping electrodes. Ion packets in proximity to a detection plate interact with the electrons in that plate. The current between the two detection plates, called the mirror current, is amplified, converted into a voltage, and measured at regular time intervals. This measurement is called the transient, and its Fourier transform yields the cyclotron frequencies of the ions. Frequencies can then be converted into $\mathrm{m} / \mathrm{z}$ ratios.
Because the natural radius of ions over the mass range of analytical interest $(<1 \mathrm{~mm})$ is very small compared to the radius of the ICR cell $(3 \mathrm{~cm})$, and because the ions' initial phases are random (i.e. incoherent), causing destructive interference of any tiny electrical signals they do generate, the mirror current is vanishingly small when ions are at rest in the ICR cell. Therefore, ions are resonantly excited with a radiofrequency voltage applied to the excitation plates, as shown in Fig. 2. Typically, the mass range of interest is between $\mathrm{m} / \mathrm{z}, 100$ and $\mathrm{m} / \mathrm{z}, 3000$. The corresponding frequency range is between $2 \mathrm{MHz}$ and $35 \mathrm{kHz}$ (which is $\sim 100 \mathrm{Da}$ per charge and $\sim 3000$ Da per charge respectively at $7 \mathrm{~T}$ ). The radiofrequency voltage is therefore a broadband excitation (Comisarow and Marshall 1974). The cyclotron radius of the ions at the end of the excitation is proportional to the amplitude and the duration of the radiofrequency voltage and does not depend on $\mathrm{m} / \mathrm{z}$ ratio (Gorshkov and Nikolaev 1993).

The two methods to generate excitation voltages are frequency sweep excitation and stored waveform inverse Fourier transform (SWIFT) (Comisarow and Marshall 1974; Wang et al. 1986). Frequency sweep excitation consists in applying a sinusoidal voltage with a variable frequency that sweeps through the frequency range. In SWIFT, the inverse Fourier transform of the desired frequency envelope is calculated to generate the time domain waveform that is to be applied to the excitation plates. A phase function can be calculated to spread out the signal in the time domain and reduce the maximum voltage on the excitation plates (Guan and McIver 1990).

After excitation, ions form coherent packets which rotate at their reduced cyclotron frequency (Nikolaev et al. 2007), which corresponds to the frequency of the cyclotron motion of the ions plus the frequency of the motion caused by the trapping potential. Ion packet coherence enables mirror current detection between the detection plates, because at each moment during detection all ions of a given $\mathrm{m} / \mathrm{z}$ ratio are closer to one detection plate than the other. The mirror current is measured at regular intervals (shorter than twice the period of the orbit of the fastest ion), converted into a

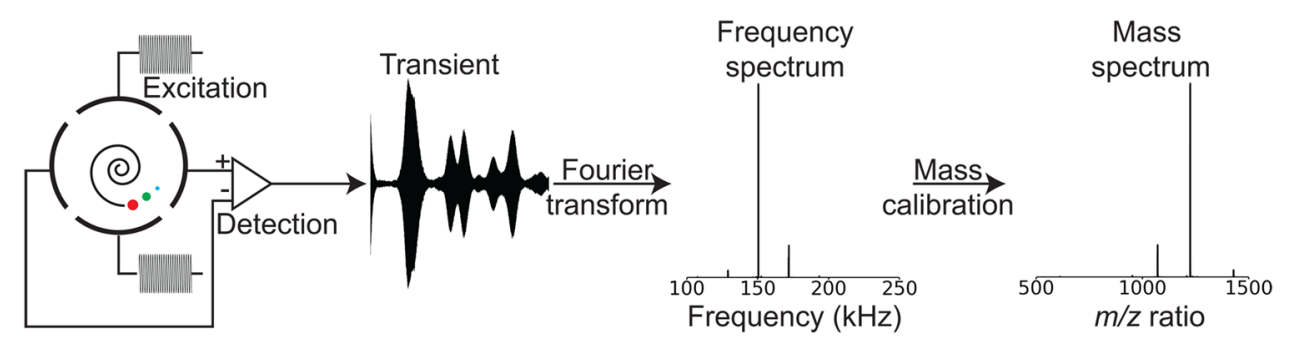

Fig. 2 Acquisition and processing of a mass spectrum with an FT-ICR mass spectrometer. The mirror current is measured between the detection electrodes, amplified and converted into a voltage, and acquired. A FT yields the frequency spectrum, and a calibration by the frequency-to-mass conversion yields the mass spectrum 
voltage and amplified (Comisarow 1978; Jerri 1977). The resulting measurement is called the transient (see Fig. 2).

Ion packet coherence can be destroyed by Coulombic repulsion, which limits the number of ions in the ICR cell, or by collisions with residual background gas in the ICR cell (Aizikov et al. 2009; Boldin and Nikolaev 2009). To maintain ion packet coherence as long as possible in the ICR cell, the pressure in the ICR cell needs to be between $10^{-10}$ and $10^{-9}$ Torr at all times. In these vacuum conditions, ion packet coherence can last longer than $1 \mathrm{~s}$ and up to several minutes with careful experimental tuning.

Fourier transformation of the transient leads to a frequency spectrum showing peaks at the frequencies of the ions (see Fig. 2). Long transients of over $1 \mathrm{~s}$ lead to frequency measurement precisions of lower than $1 \mathrm{~Hz}$ (Nikolaev et al. 2011). For an ion at $\mathrm{m} / \mathrm{z} 400$ rotating in a magnetic field of $12 \mathrm{~T}$, the cyclotron frequency is $460 \mathrm{kHz}$. This leads to resolving power at $\mathrm{m} / \mathrm{z} 400$ that easily exceeds 230,000 (in $1 \mathrm{~s}$ ) and mass accuracies better than $1 \mathrm{ppm}$ (Nikolaev et al. 2012). Provided that the ion number is kept low enough that Coulombic repulsion amongst the ions is minimal, the main factors determining the resolving power in an FT-ICR mass spectrum are the magnetic field, the transient duration (itself limited by the magnetic field), the pressure inside the ICR cell, and the acquisition time (Amster 1996; Marshall et al. 1998).

The mass spectrum is obtained by converting the reduced cyclotron frequencies into $\mathrm{m} / \mathrm{z}$ ratios. The high resolving power of the frequency spectrum leads to high resolving powers in the mass spectra as well. In a mass spectrum, because the $m / z$ ratio is in first approximation inversely proportional to the frequency, the resolving power decreases with increase in $\mathrm{m} / \mathrm{z}$ ratios. FT-ICR MS is considered to be the mass analyser with the highest resolution and mass accuracy of all (Marshall and Hendrickson 2008; Valeja et al. 2011; Wei et al. 2016). Nevertheless, in the Orbitrap mass analyser, the resolving power decreases with the square root of the $\mathrm{m} / \mathrm{z}$ ratio, which means that at high $\mathrm{m} / \mathrm{z}$ ratios, Orbitraps may have higher resolving powers than FT-ICR mass analysers (Denisov et al. 2012).

A first approximation of the frequency-to-mass conversion can be obtained using Eq. 1. The use of a trapping voltage between the two trapping electrodes changes the frequency-to-mass conversion because the measured frequency is the reduced cyclotron frequency instead of the cyclotron frequency. Linear or quadratic functions are therefore most often used to convert frequencies into $\mathrm{m} / \mathrm{z}$ ratios (Francl et al. 1983; Ledford et al. 1984; Shi et al. 2000). In most FT-ICR mass spectrometers, the instrument is calibrated daily using a standard compound mixture to correct the effect of the magnetic field drift (typically of the order of a ppb/day). Internal calibration of mass spectra uses the measured frequency of the peaks at their centroid and the theoretical $\mathrm{m} / \mathrm{z}$ ratio of the compounds they are assigned to. A typical internal calibration uses a quadratic frequency-tomass conversion:

$m / z=\frac{A}{\omega^{2}}+\frac{B}{\omega}+C$,

in which $\mathrm{m} / \mathrm{z}$ is the theoretical $\mathrm{m} / \mathrm{z}$ ratio, $\omega$ the measured frequency of the peak at its centroid, and $A, B$, and $C$ are constants that are calculated using a quadratic regression (Ledford et al. 1984). If the assignments of the peaks are correct, then internal calibration reduces the mass accuracies of the peak assignments.

For tandem mass spectrometry, there are multiple isolation methods in an FT-ICR mass spectrometer. Because of the pressure constraints, the ion source is external to the ICR cell and ions are transported through ion optics to the ICR cell. The ion optics often consist of electrodes that form a multipolar electric field, whose voltages can be manipulated to enable trajectory stability for ions within a mass window of $1 \mathrm{Da}$ or less, therefore enabling ion isolation in the front end of the FT-ICR mass spectrometer (Gerlich 1992; Jebanathirajah et al. 2005; O'Connor et al. 2006). Ion isolation can also be achieved in the ICR cell by exciting all ions except the ion of interest radially up to the electrodes with a resonant excitation. Both a frequency sweep that skips the frequencies of the ions of interest (CHEF) and a SWIFT excitation with a notched frequency envelope can be used for this purpose (de Koning et al. 1997; McDonald et al. 2003; O'Connor et al. 1996; O'Connor and McLafferty 1995).

The most popular fragmentation method is collisioninduced dissociation (CID, also called collisionally activated dissociation, or CAD), which consists in non-elastic collisions between ions and a neutral gas. During each collision, part of the kinetic energy is converted into internal energy, which leads to the fragmentation of the bonds with the lowest dissociation energy in the ion (Sleno and Volmer 2004). The main parameters used to control the degree of dissociation are the nature of the neutral gas (the most often used gas is argon), its pressure in the collision cell, and the kinetic energy of the ions in the collision cell.

Infrared multiphoton dissociation (IRMPD) relies on the absorption of infrared photons by ions (Little et al. 1994). The IR photons are often generated by a $\mathrm{CO}_{2}$ laser with a $10.6 \mu \mathrm{m}$ wavelength (Garnier et al. 2009). The absorption of IR photons increases the internal energy of the ions, and the bonds with the lowest energy rearrangements are dissociated (Ramaswamy et al. 1981). Another photon-based fragmentation method is ultraviolet photodissociation (UVPD), in which a UV photon generated by an NdYAG laser ( $266 \mathrm{~nm}$ or $213 \mathrm{~nm})$ or an excimer laser $(193 \mathrm{~nm})$ is absorbed by ions (Agarwal et al. 2011; Madsen et al. 2010; Racaud et al. 2009). In both IRMPD and UVPD, the fragmentation efficiency depends on the presence of a chromophore in the ion. 
The first electron-based fragmentation method is electron capture dissociation (ECD), in which multiply charged positive ions capture one or more electrons generated by an electron gun, which destabilizes the structure of the ion and leads to fragmentation (Kruger et al. 1999; Zubarev et al. 1998). However, because of mass differences between ions and electrons and the voltages typically applied to ion trap electrodes, electrons and ions can only be trapped simultaneously in a high magnetic field, which means that ECD is most practicable in FT-ICR mass spectrometers. Although ECD can only be used with positive ions with multiple charges, other electron-based fragmentation methods have been developed to achieve fragmentation of singly charged positive ions (electron-induced dissociation, EID, or electron impact excitation of ions from organics, or EIEIO) (Cody and Freiser 1979; Haselmann et al. 2002; Mosely et al. 2011; Wei et al. 2013; Wills and O'Connor 2014). To fragment negative ions, electron-detachment dissociation (EDD) and negative ion ECD (niECD) have been developed (Budnik et al. 2001; Kaczorowska and Cooper 2008; Song and Hakansson 2012).

The fragmentation methods listed in the previous paragraph can be performed inside the ICR cell. Laser-based dissociation methods like IRMPD and UVPD are performed at the centre of the ICR cell. Electron-based dissociation methods have a fragmentation zone that depends on the shape of the electron gun. For example, in the case of a hollow cathode, the shape of the fragmentation zone has a maximum at a non-zero radius, but has significant fragmentation efficiency at the centre of the ICR cell (Tsybin et al. 2003). Because of the pressure constraints in the ICR cell, fragmentation methods that require gas, like CAD or electron-transfer dissociation (ETD), are mostly performed in a collision cell in the front end of the mass spectrometer (Syka et al. 2004).

Being able to use various fragmentation methods to perform tandem mass spectrometry can yield complementary structural information. For example, in the case of peptides and proteins, IRMPD preferentially fragments post-translational modifications such as glycosylations and phosphorylations, whereas ECD preferentially fragments the amino acid backbone (Hakansson et al. 2001). Fragmenting a peptide or a protein with both ECD and IRMPD can therefore increase the amount of structural information available on them.

Because of its transient duration, the duty cycle of an FTICR mass spectrometer (i.e. the time necessary to acquire a single mass spectrum) is of the order of $1 \mathrm{~s}$. FT-ICR mass spectrometers can therefore be coupled with chromatographic methods. However, DIA methods such as PAcIFIC, SWATH, or MS ${ }^{\mathrm{E}}$, depend on mass analysers with duty cycles of the order of $1 \mathrm{~ms}$. Coupling these DIA methods with an FT-ICR mass spectrometer would lead to very long experiments, high sample consumption, and very large datasets.

\section{Principle of two-dimensional mass spectrometry}

In 1984, Marshall et al. demonstrated the reversibility of resonant excitation inside the ICR cell. In a first experiment, ions were excited with a single frequency resonant excitation with a constant amplitude and increasing duration (Marshall et al. 1984). Since the radius of the ions after excitation increased, the intensity of the peak in the mass spectrum increased until the ion radius reached the radius of the ICR cell. In a second experiment, the phase of the resonant excitation was switched by $180^{\circ}$ after $1 \mathrm{~ms}$, by inversing the sign of the applied voltage. A decrease of the peak was observed until an excitation duration of $2 \mathrm{~ms}$, when the peak intensity was zero. In the first experiment, the ion packet remained closest to the excitation plate with the attractive potential, which caused the radius to increase. In the second experiment, after the phase switch, the ions were closest to the excitation plate with the repelling voltage, which caused the radius to decrease. After $2 \mathrm{~ms}$, the ions were back at the centre of the ICR cell, and therefore undetectable. This experiment proved that ion excitation was reversible in the ICR cell.

In 1987, Pfändler et al. proposed a pulse sequence for two-dimensional mass spectrometry based on Marshall's conclusions (Pfändler et al. 1987). This pulse sequence is shown in Fig. 3a. The first pulse $\mathrm{P}_{1}$ excites ions from the centre of the ICR cell to a radius $r_{1}$. During the delay $t_{1}$, the phase of the cyclotron motion of the ions increases by $\omega_{\text {ICR }} \times t_{1}$, in which $\omega_{\text {ICR }}$ is the cyclotron motion of the ions. After $t_{1}$, a pulse $\mathrm{P}_{2}$ which is identical to $\mathrm{P}_{1}$ is applied. The frequency range of $\mathrm{P}_{1}$ and $\mathrm{P}_{2}$ needs to cover the cyclotron frequency range of all the precursor ions of interest in the sample.

If $\varphi_{\text {ion }}$ is the phase of the ion trajectory in the ICR cell in polar coordinates around the axis at the centre of the ICR cell and $\varphi_{\mathrm{RF}}$ is the phase of the RF voltage, then the difference $\Delta \varphi$ between the two at the end of $\mathrm{P}_{2}$ can be expressed as:

$\Delta \varphi=\varphi_{\text {ion }}-\varphi_{\mathrm{RF}}=\left(\omega_{\mathrm{ICR}}-\omega_{\mathrm{N}}^{\mathrm{RF}}\right) t_{1}+\Delta \psi$,

in which $\omega_{\mathrm{N}}^{\mathrm{RF}}$ is the last frequency in the $\mathrm{P}_{1}$ pulse and $\Delta \psi$ is a constant that has been shown by Pfändler et al. to only depend on the characteristics of $\mathrm{P}_{1}$ and $\mathrm{P}_{2}$ (Pfändler et al. 1988). The term $-\omega_{\mathrm{N}}^{\mathrm{RF}}$ is needed when a continuous phase pulse generator is used (this is the case for pulse generators in Bruker FT-ICR mass spectrometers, which are the most popular commercial instruments). In this case the generator keeps its central clock oscillating at the lowest generated frequency and generates the $\mathrm{P}_{2}$ pulse with the accumulated phase of this frequency over the $t_{1}$ period. A non-continuous phase generator may cause other difficulties which may hamper altogether the possibility of recording the modulation during $t_{1}$. The radius at the end of $\mathrm{P}_{2}$ can be expressed as: 
Fig. 3 a Pulse sequence for a 2D MS experiment. The encoding sequence modulates the radius of the ions according to their cyclotron frequency and the delay $t_{1}$. The radiusdependent fragmentation then modulates the abundance of the precursors and all ions are excited and detected.

b Evolution of ion cyclotron radii during a 2D MS experiment. According to the product of their cyclotron frequency and the delay $t_{1}$, the ion packets have different radii at the end of the encoding sequence. $\mathbf{c}$ Fragmentation efficiency at different cyclotron radii. The cyclotron radii of the ions after the encoding sequence determines the fragmentation efficiency. d Overlap between the fragmentation zone and the radius modulation. There is an optimal overlap between the fragmentation zone and the amplitude of the cyclotron radius modulation where multiple $\omega_{1}$ harmonics are minimized (a) Pulse sequence for two-dimensional mass spectrometry

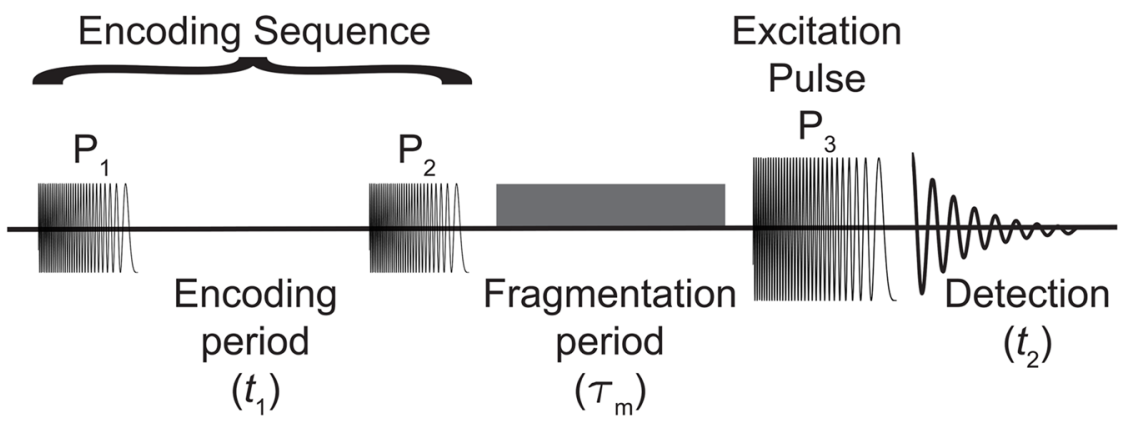

(b) Evolution of ion cyclotron radii

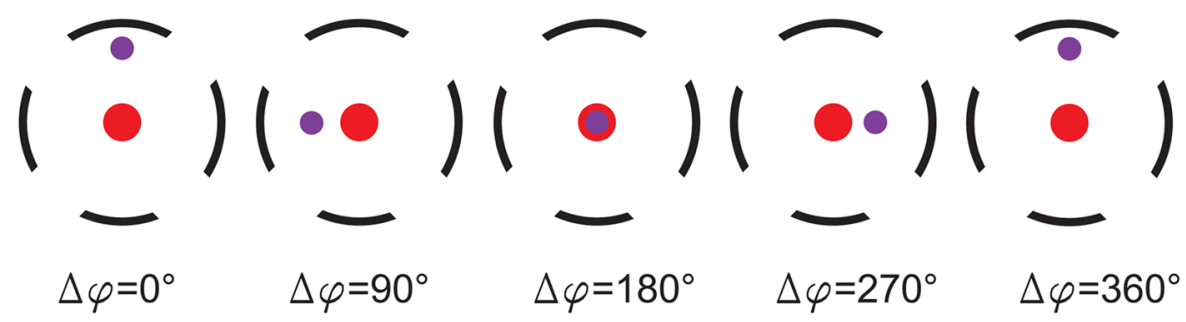

(c) Fragmentation efficiency at different ion cyclotron radii

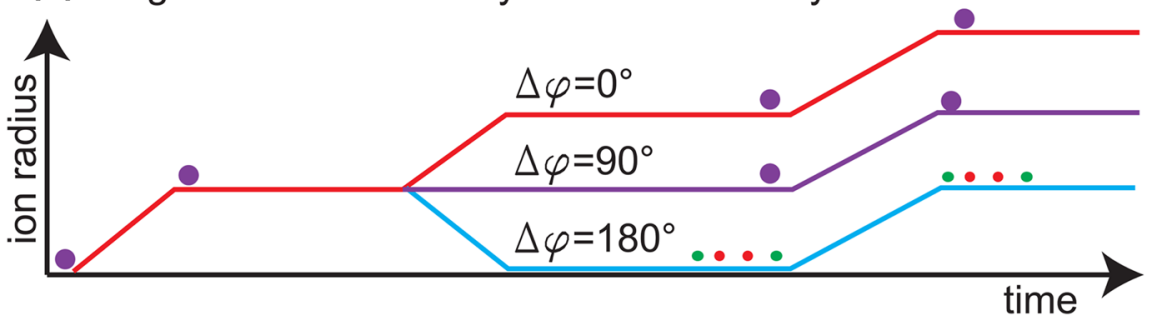

(d) Overlap between the fragmentation zone and the cyclotron radius modulation

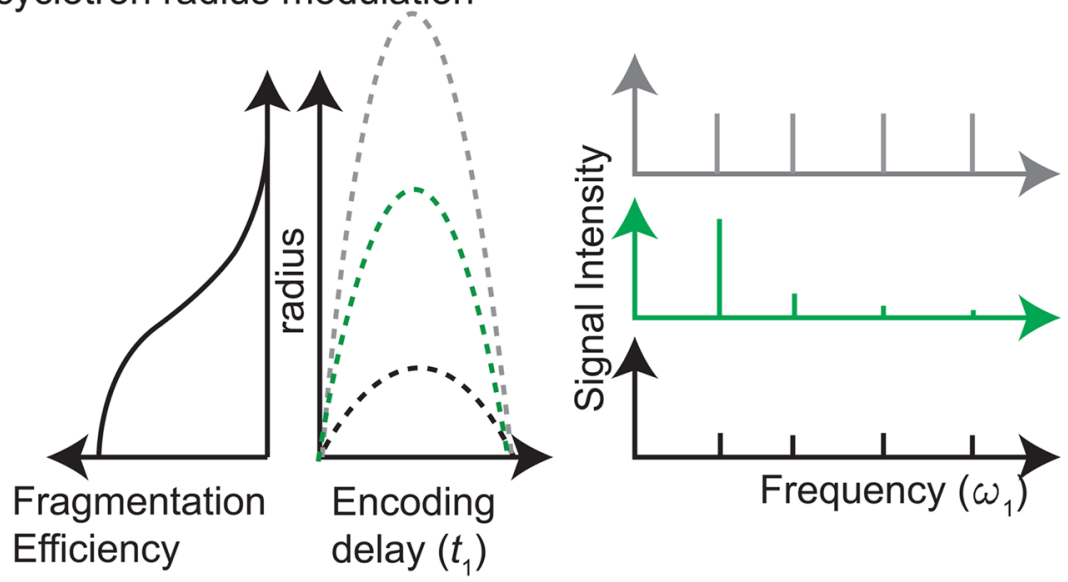

$r\left(t_{1}\right)=r_{1} \sqrt{2\left(1+\cos \omega_{A}\left(t_{1}-T\right)\right)}$,

in which $\omega_{\mathrm{A}}=\omega_{\mathrm{ICR}}-\omega_{\mathrm{N}}^{\mathrm{RF}}, r_{1}$ is the radius of the ions at the end of $\mathrm{P}_{1}$, and $T$ is the duration of $\mathrm{P}_{1}$ (Guan and Jones 1989).
Figure $3 \mathrm{~b}$ shows the cyclotron radii of the ions at the end of $\mathrm{P}_{2}$ for different values of $\Delta \varphi$ (cf. Eq. 3). If $\Delta \varphi=0^{\circ}$, then ions are attracted by the excitation electrode they are closest to during resonant excitation in $\mathrm{P}_{2}$. The ion packet is therefore excited to double their radius from what it was 
at the end of $\mathrm{P}_{1}$. If $\Delta \varphi=180^{\circ}$, then ions are repelled by the excitation electrode they are closest to during resonant excitation in $\mathrm{P}_{2}$. The effect of $\mathrm{P}_{2}$ therefore is that the ion packet is de-excited and goes back to the centre of the ICR cell. If $\Delta \varphi=90^{\circ}$ or $\Delta \varphi=270^{\circ}$, then the ion packet is at equal distance from either excitation electrode during resonant excitation in $\mathrm{P}_{2}$, and $\mathrm{P}_{2}$ has no effect on the ion packet radius. When $\Delta \varphi=360^{\circ}$, the situation is identical to the $\Delta \varphi=0^{\circ}$ situation. After radius modulation of the precursor ions, a fragmentation mode with radius-dependent fragmentation efficiency is applied for a duration of $\tau_{\mathrm{m}}$. Figure $3 \mathrm{c}$ shows how the ion packet radius at the end of $\mathrm{P}_{2}$ affects fragmentation efficiency. The diagram in Fig. $3 \mathrm{c}$ is reminiscent of coherence transfer pathway diagrams used in nuclear magnetic resonance spectroscopy (NMR), which display the coherence order explored by the spin system while pulses are applied to the sample (Bodenhausen et al. 1984). In NMR, the quantum state can only take discrete values, which can also be negative. However, both diagrams share many properties: the initial state is always at zero; only pulses may modify the level value; this value describes the system state; the final state, where acquisition of the signal occurs, has to be on a special value ( -1 in NMR vs. a narrow range of radii in 2D FT-ICR).

For fragmentation methods like ion-molecule reactions or $\mathrm{CAD}$, the fragmentation efficiency increases with the internal energy of the precursor ions after collision, i.e. with their kinetic energy when collisions happen. In the ICR cell, after radius modulation in a 2D FT-ICR MS experiment, the kinetic energy of the precursor ions, and therefore their fragmentation efficiency, is proportional to $r^{2}\left(t_{1}\right)$ (Bensimon et al. 1989).

In IRMPD, the laser is aligned with the axis at the centre of the ICR cell. Ions absorb multiple photons which increase their kinetic energy until fragmentation happens. Fragmentation efficiency is therefore proportional to photon density and decreases when precursor ion radii increase. In IRMPD, occurrences of secondary fragmentation (i.e. fragment ions that absorb photons and then fragment) need to be considered (van Agthoven et al. 2011b). In ECD, the fragmentation efficiency depends on the electron density in the ICR cell, which depends on the shape of the electron emitter. Broadly speaking, the electron density is largest close to the centre of the ICR cell, and fragmentation efficiency decreases when the precursor ion radius increases (van Agthoven et al. 2012).

Because the presence of gas in the ICR cell destroys ion packet coherence, $\mathrm{CAD}$ is not a fragmentation method that is compatible with 2D MS. IRMPD and ECD do not require any gas, and so they are the most used. When $\Delta \varphi$ is at a value (e.g. $0^{\circ}$ or $90^{\circ}$, or red and purple lines in Fig. 3c, for IRMPD) where the ions are outside of the fragmentation zone (red zone in Fig. 3b), then no fragmentation occurs during $\tau_{\mathrm{m}}$. When $\Delta \varphi$ is at a value (e.g. close to $180^{\circ}$, or blue line in Fig. 3c, for IRMPD) where the ions are within the fragmentation zone, then fragmentation occurs during $\tau_{\mathrm{m}}$. After $\tau_{\mathrm{m}}$, all ions (unfragmented precursors and fragments) are excited and detected. A transient is recorded for each value of $t_{1}$. The precursor ions are modulated in and out of the fragmentation zone at different frequencies, and the fragment ion abundance therefore carries the modulation frequency of the precursor ions.

In the 2D MS pulse sequence, the ion radius modulation $r_{1}$ has to be optimized according to the size of the fragmentation zone. Figure $3 \mathrm{~d}$ shows how the overlap between the radius modulation and the fragmentation zone affects the $2 \mathrm{D}$ mass spectrum. If the radius modulation is small compared to the fragmentation zone (black lines in Fig. 3d), then there is very little modulation of the ion abundance (precursor and fragment), and the intensity of the peaks in the spectrum after Fourier transformation (FT) according to $t_{1}$ is very low. If the radius modulation is large compared to the fragmentation zone (grey line in Fig. 3d), then the evolution of the ion abundance shows periodic sharp peaks, and the peaks in the spectrum after FT according to $t_{1}$ will have intense harmonics. If the radius modulation has the same range as the fragmentation zone (green line in Fig. 3d), then the evolution of the ion abundance is close to a sinusoid, and the peaks in the spectrum after FT according to $t_{1}$ is at its maximum intensity and the harmonics are at low intensity (van Agthoven et al. 2014).

At the end of $\tau_{\mathrm{m}}$, both precursor and fragment ions coexist in the ICR cell at different radii. If the fragmentation method used during $\tau_{\mathrm{m}}$ does not require pulsing a gas into the ICR cell (e.g. IRMPD or ECD), then the pressure in the ICR cell is sufficiently low that issues of coherence loss of the ion packet do not occur. The abundance of the fragment ions depends on the radius of the precursor ions at the end of $\mathrm{P}_{2}$. As a result, following Eq. 3, they are modulated according to $t_{1}$ and $\omega_{\mathrm{A}}=\omega_{\mathrm{ICR}}-\omega_{\mathrm{N}}^{\mathrm{RF}}$, i.e. the cyclotron frequency of their precursors. When precursor ions are fragmented, their abundance decreases, which leads to a modulation of both precursor ion radii and precursor ion abundance according to $t_{1}$ and according to $\omega_{\mathrm{A}}=\omega_{\mathrm{ICR}}-\omega_{\mathrm{N}}^{\mathrm{RF}}$ at the end of $\tau_{\mathrm{m}}$. Precursor ion abundances follow a modulation which depends on Eq. 3, while fragment ion abundances follow $1-r\left(t_{1}\right)$ (see Eq. 4).

After fragmentation, all ions in the ICR cell are excited by a third pulse $\mathrm{P}_{3}$ (see Fig. 3a, b), prior to detection. The frequency range of $\mathrm{P}_{3}$ needs to cover the cyclotron frequencies of all the fragment ions of interest. In one-dimensional FT-ICR MS, ions are excited from the centre of the ICR cell to the optimal radius for detection before detection. In 2D FT-ICR MS, ions are within a range of $2 r_{1}$ at the start of $\mathrm{P}_{3}$. However, if $2 r_{1}$ is significantly smaller than the radius of ions after $\mathrm{P}_{3}$, then the ions in the ICR cell at the end of $\tau_{\mathrm{m}}$ 
can be assumed to be at the centre of the ICR cell and the effect of $\mathrm{P}_{3}$ can be assumed to be the same for all ions (van Agthoven et al. 2014).

In the 2D FT-ICR MS experiment, each iteration of the pulse sequence contains the following events:

- Ionization, external ion accumulation, and ion transfer to the ICR cell (0.05-1 s).

- $\mathrm{P}_{1}-t_{1}-\mathrm{P}_{2}$ encoding sequence (typically a few $\mathrm{ms}$ ).

- Fragmentation (10 ms-1 s).

- $\mathrm{P}_{3}$ excitation (20 ms).

- Transient acquisition (0.5 s).

Each iteration of the pulse sequence lasts between 0.6 and $2 \mathrm{~s}$, approximately. The most important factors in the duration of an experiment are the number of scans, the ion accumulation, the fragmentation period, and the duration of the transient. With a continuous ion source such as electron impact ionization (EI), electrospray (ESI), nanospray (nanoESI), or atmospheric pressure photoionization (APPI), the amount of sample consumed is proportional to the duration of the experiment (van Agthoven et al. 2011b, 2015). For punctual ion sources such as matrix-assisted laser desorption ionization (MALDI), the amount of sample consumed only depends on the number of laser shots accumulated.

The acquisition of a dataset for a 2D FT-ICR MS experiment is set at regularly incremented values of $t_{1}$. The number of data points and their time increment in each dimension determine the mass range for the precursors and the fragments, the total experimental time, as well as the resolving power and signal-to-noise ratio of each peak. For each value of $t_{1}$, a transient is measured, acquired, and saved to obtain a 2D dataset. The dataset acquired from a $2 \mathrm{D}$ FT-ICR MS experiment can be expressed as $S\left(t_{1}, t_{2}\right)$, where $t_{1}$ is the duration of the delay in the sequence, during which the precursor ions have been let to evolve, and $t_{2}$ corresponds to the transient detection period. Both $t_{1}$ and $t_{2}$ have similar roles in the expression of the signal; however, they correspond to very different actions. Each value of $t_{1}$ corresponds to a measure of a transient, while $t_{2}$ is the classical time during the measurement of the transient (i.e. $t_{1}$ is a delay and $t_{2}$ is the date at which each transient point was measured).

To process a dataset from a two-dimensional mass spectrometry experiment, the FT of each transient according to $t_{2}$ is calculated (and can be expressed as $S\left(t_{1}, \omega_{2}\right)$ ), followed by the FT of the signal at each frequency in the resulting spectra according to $t_{1}$ (and can be expressed as $S\left(\omega_{1}, \omega_{2}\right)$ ). The $\omega_{1}$ frequency measures the cyclotron frequency of the precursor ions and the $\omega_{2}$ frequency measures the cyclotron frequency of the fragment ions. Therefore, the $\omega_{1}$ dimension is called the precursor ion dimension and the $\omega_{2}$ dimension is called the fragment ion dimension (van Agthoven et al. 2013). One program that has been developed with $2 \mathrm{D}$ MS in mind is SPIKE (Spectroscopy Processing Innovative KErnel), an open source Python package dedicated to Fourier spectroscopies (Chiron et al. 2016).

To optimize the quality of the 2D spectrum, each transient, both in $t_{1}$ and $t_{2}$, is apodized and subsequently zerofilled. Zerofilling consists in extending the experimental transient with null values. Without zerofilling, the FT of an $\mathrm{N}$ real points dataset produces only N/2 useful information, and loses information. By at least doubling the size of the transient (i.e. "zerofilling once"), all spectral information is recovered. In 2D MS, zerofilling has to be performed at least once along each dimension, which means that the size of the dataset is temporarily multiplied by 4 during the processing (Marshall and Verdun 1990).

Apodization is performed to compensate for the fact that a dataset has a finite duration. The end of a dataset can be modelled by multiplying the dataset by a box function, which leads to convoluting the spectrum by a $\sin (x) / x$ (also called $\operatorname{sinc}(x))$ function and therefore "wriggles" or "feet" distorting each peak in the spectrum. Apodization corresponds to the multiplication of each transient by a windowing function which smooths the ends of the transient and minimizes the wriggles on the sides of each peak. In 2D FT-ICR MS processing, apodization is performed on each transient before FT according to $t_{2}$, and then on $S\left(t_{1}, \omega_{2}\right)$ before FT according to $t_{1}$ (Marshall and Verdun 1990). Many apodization windows exist currently in SPIKE. Apodization by a Kaiser $(\beta)$ window seems to give the best results, with $\beta=3.5$ along $t_{2}$ and $\beta=5$ along $t_{1}$ (Kuo 1966).

Every data point in $S\left(t_{1}, t_{2}\right)$ is real. After the first FT according to $t_{2}$, the data points of the resulting dataset $S\left(t_{1}, \omega_{2}\right)$ are complex. Because the evolution in $t_{1}$ comes from a different phenomenon than the modulation in $t_{2}$, the amplitude is modulated (see Eq 4). In consequence, the real and imaginary components of $S\left(t_{1}, \omega_{2}\right)$ have to be transformed independently as two independent real series. After this second FT according to $t_{1}$, the data points of the resulting dataset $S\left(\omega_{1}, \omega_{2}\right)$ have four components: $\operatorname{RR}\left(\omega_{1}, \omega_{2}\right), \operatorname{RI}\left(\omega_{1}, \omega_{2}\right)$, $\operatorname{IR}\left(\omega_{1}, \omega_{2}\right)$, and $\operatorname{II}\left(\omega_{1}, \omega_{2}\right) . S\left(\omega_{1}, \omega_{2}\right)$ is expressed as a hypercomplex number (Delsuc 1988; Ernst et al. 1987):

$S\left(\omega_{1}, \omega_{2}\right)=\operatorname{RR}\left(\omega_{1}, \omega_{2}\right)+i \operatorname{RI}\left(\omega_{1}, \omega_{2}\right)+j \operatorname{IR}\left(\omega_{1}, \omega_{2}\right)+k \operatorname{II}\left(\omega_{1}, \omega_{2}\right)$,

With

$i^{2}=j^{2}=-1$

$k^{2}=1$

The hypercomplex values $i, j$, and $k$ follow these rules:

$i \cdot j=j \cdot i=k$

$i \cdot k=k \cdot i=-j$

$j \cdot k=k \cdot j=-i$ 
The magnitude mode spectrum $M\left(\omega_{1}, \omega_{2}\right)$ can be obtained using the hypercomplex modulus for each data point:

$$
\begin{aligned}
& M\left(\omega_{1}, \omega_{2}\right) \\
& =\sqrt{\operatorname{RR}\left(\omega_{1}, \omega_{2}\right)^{2}+\operatorname{RI}\left(\omega_{1}, \omega_{2}\right)^{2}+\operatorname{IR}\left(\omega_{1}, \omega_{2}\right)^{2}+\operatorname{II}\left(\omega_{1}, \omega_{2}\right)^{2}}
\end{aligned}
$$

The Nyquist frequencies, which correspond to the highest frequencies detected, are determined by the following equations:

$f_{N 1}=\frac{1}{2 \Delta t_{1}}$

and

$f_{N 2}=\frac{1}{2 \Delta t_{2}}$

in which $f_{N 1}$ is the Nyquist frequency in the precursor ion dimension, $\Delta t_{1}$ the increment in $t_{1}, f_{N 2}$ the Nyquist frequency in the fragment ion dimension, and $\Delta t_{2}$ the increment in the acquisition of the transient $t_{2}$. The $f_{N 1}$ frequency determines the lowest precursor $\mathrm{m} / \mathrm{z}$ ratio and the $f_{N 2}$ frequency the lowest fragment $\mathrm{m} / \mathrm{z}$ ratio.

The lowest frequency in excitation pulses is of the order of several $10 \mathrm{kHz}$. For example, on an FT-ICR mass spectrometer with a $12 \mathrm{~T}$ magnet, an excitation pulse for a mass range with a maximum $m / z$ ratio of $m / z, 3000$, the lowest frequency is $56.4 \mathrm{kHz}$. Any ion with a cyclotron frequency below this value does not get excited. In the 2D FT-ICR MS pulse sequence, the radius of such an ion does not get modulated. As a result, the lowest useful frequency in a 2D mass spectrum is determined by the lowest frequency in the excitation pulse $\left(\mathrm{P}_{1}\right.$ and $\mathrm{P}_{2}$ for precursor ions, and $\mathrm{P}_{3}$ for fragment ions).

An additional correction step is required during the processing of the vertical axis because of the frequency correction $-\omega_{\mathrm{N}}^{\mathrm{RF}}$ term which appears in Eq. 3 as a correction of the continuous phase pulse generator. This additional frequency produces a phase modulation of the precursor signal along $t_{1}$ superimposed to the amplitude modulation produced by the fragmentation event and described by Eq. 4. Uncorrected, this modulation splits all frequencies by an offset $\pm \omega_{\text {off }}$ with $\omega_{\text {off }}=\omega_{\mathrm{N}}^{\mathrm{RF}}$ in the precursor ion dimension.

For this reason, a digital demodulation of each signal as a function of $t_{1}$ is performed in the data processing by multiplying each column in the dataset by $e^{i\left(\frac{\pi}{2} \omega_{\text {off }} t_{1}-\frac{\pi}{2}\right)}$ before the FT according to $t_{1}$. The removal of this frequency splitting allows a $\sqrt{ } 2$ gain in signal-to-noise ratio. As a further consequence, unmodulated signals (such as non-fragmenting ions) appear at the $\omega_{\text {off }}$ frequency rather than at the zero frequency, and appear in the final spectrum as a strong horizontal line, located at $\omega_{\text {off }}$, i.e. at the highest excited mass. In the same manner, the highest (Nyquist) measured frequency is lowered by the same amount. Frequency-to-mass conversion following Eq. 2 can be applied to both dimensions of the 2D mass spectrum.

The resolving power at $\mathrm{m} / \mathrm{z}, 400$ in a $2 \mathrm{D}$ mass spectrum depends on the intrinsic resolution of the instrument (i.e. the magnetic field), the experimental resolution (i.e. mostly the pressure in the ICR cell), and the measurement (i.e. the duration of the acquisition and the increment in delay or measurement time). The resolving power in a $2 \mathrm{D}$ mass spectrum can be separated into two values: the vertical precursor ion resolving power and the horizontal fragment ion resolving power. In both dimensions, the resolving power increases with the magnetic field and decreases when the pressure in the ICR cell increases. Because in both dimensions the spectrum is obtained using an FT, the resolving power is proportional to the frequency and inversely proportional to the $\mathrm{m} / \mathrm{z}$ ratio. The resolving power in each dimension is proportional to the maximum values of $t_{1}$ and $t_{2}$ (i.e. total acquisition time). In the fragment ion dimension, the resolving power indicates the capacity to separate the $\mathrm{m} / \mathrm{z}$ ratios of fragment ions. In the precursor ion dimension, the resolving power indicates the accuracy of the correlation between precursor and fragment ions (van Agthoven et al. 2016).

At a given resolving power, the number of data points can be decreased by increasing the low mass limit (i.e. decreasing the Nyquist frequency). For a given acquisition time and number of data points, a choice can be made to favour the resolving power in one dimension or the other. For example, in bottom-up proteomics, the mass spectrum of the precursor ions is very dense and separation in the vertical precursor ion dimension is crucial. However, the fragment ion scans are typically less dense. A large number of data points in the vertical precursor ion dimension (e.g. 4096) is therefore more important than a large number of data points in the horizontal fragment ion dimension (e.g. 262,144). In top-down proteomics, the complexity of the fragment ion scans demands a large number of data points in the horizontal fragment ion dimension (e.g. 4,194,304) (Floris et al. 2016). Finally, there is one degree of freedom possible with non-uniform sampling, where skipping data points along $t_{1}$ can significantly enhance the resolving power in the vertical precursor dimension without increasing the total acquisition time (Bray et al. 2017).

The resolving power can also be seen as the maximum number of independent signals that can be resolved in a spectrum. The maximum number of signals resolvable in 2D MS is the product of both resolving powers of precursor and fragment axes. This third value that we can call $2 \mathrm{D}$ resolving power is a large number, usually well over $10^{7}$. 


\section{Interpretation of two-dimensional mass spectra}

After data processing, the 2D mass spectrum can be viewed in the $\mathrm{m} / \mathrm{z}$ ratio domain. Figure 4 shows the $2 \mathrm{D}$ mass spectrum of a tryptic digest of bovine serum albumin (BSA) with IRMPD fragmentation. Fragment ion $\mathrm{m} / \mathrm{z}$ ratios can be read on the horizontal axis and precursor ion $\mathrm{m} / \mathrm{z}$ ratios can be read on the vertical axis. Each peak corresponds to a fragmentation (van Agthoven et al. 2013).

The 2D mass spectrum (Fig. 4a) contains several characteristic lines. The autocorrelation line (extracted in Fig. 4b) corresponds to the modulation of the intensity of the precursor ions according to their own cyclotron frequency. The equation of the autocorrelation line is:

$(m / z)_{p}=(m / z)_{f}$

in which $(\mathrm{m} / \mathrm{z})_{\mathrm{p}}$ is the precursor axis $\mathrm{m} / \mathrm{z}$ ratio and $(\mathrm{m} / \mathrm{z})_{\mathrm{f}}$ is the fragment axis $\mathrm{m} / \mathrm{z}$ ratio. The autocorrelation line is not the mass spectrum of the precursor ions, because the intensity of the peaks depends on the modulation of the fragmentation efficiency during the ion radius modulation. If a precursor ion does not fragment, then it is not detected on the autocorrelation line, regardless of its abundance in the mass spectrum. The autocorrelation line of the 2D mass spectrum of the tryptic digest of BSA shows the tryptic peptides that have been obtained after digestion of the protein. With the autocorrelation line alone, a sequence coverage of $55 \%$ is obtained after protein database interrogation.

Each horizontal line in a 2D mass spectrum corresponds to the fragment ion scan of the precursor ion whose $\mathrm{m} / \mathrm{z}$ ratio can be read on the vertical axis. Figure $4 \mathrm{c}$ shows the fragment ion scan extracted at $\mathrm{m} / \mathrm{z} 710.3$ and shows the fragmentation pattern of the doubly charged ion of peptide ${ }^{89}$ SLHTLFGDELCK ${ }^{100}$, which enables its sequencing.

Each vertical line in a 2D mass spectrum corresponds to the precursor ion scan of the fragment ion whose $\mathrm{m} / \mathrm{z}$ ratio can be read on the horizontal axis. Figure $4 \mathrm{~d}$ shows the precursor ion scan of $\mathrm{m} / \mathrm{z} 549.27$, which corresponds to the $y_{4}$ fragment of peptide SLHTLFGDELCK. As can be seen on the precursor ion scan, this fragment has two precursors: the doubly charged ion and triply charged ion of peptide SLHTLFGDELCK.

Since each fragmentation can be characterized by a loss of mass and a loss of charge, it can be extracted in a line with the following equation (van Agthoven et al. 2016):

$(m / z)_{p}=\frac{n-p}{n}(m / z)_{f}+\frac{M}{n}$

in which $(\mathrm{m} / \mathrm{z})_{\mathrm{p}}$ is the precursor axis $\mathrm{m} / \mathrm{z}$ ratio, $(\mathrm{m} / \mathrm{z})_{\mathrm{f}}$ is the fragment axis $m / z$ ratio, $n$ is the charge of the precursor ion,
Fig. 4 a 2D IRMPD mass spectrum of bovine serum albumin tryptic digest. Each peak corresponds to a dissociation, with the $m / z$ ratio of the precursor plotted vertically and the $\mathrm{m} / \mathrm{z}$ ratio of the fragment plotted horizontally. b Autocorrelation line with assigned peaks and sequence coverage. The autocorrelation line shows the $\mathrm{m} / \mathrm{z}$ ratios of the precursor ions. c Fragment ion scan of $\mathrm{m} / \mathrm{z}$ 710.3. The fragment ion scan of each precursor ion can be extracted horizontally. d Precursor ion scan of $m / z, 549.27$.

The precursor ion scan of each fragment ion can be extracted vertically. e Neutral loss line of water by doubly charged precursors. Each neutral loss line can be extracted as a line that is parallel to the autocorrelation line. f Dissociation line for loss of lysine with one charge loss for doubly charged precursors. Each dissociation line is characterized by the charge states of the precursor and the fragment ions and by the mass that is lost (a) 2D MS of BSA tryptic digest

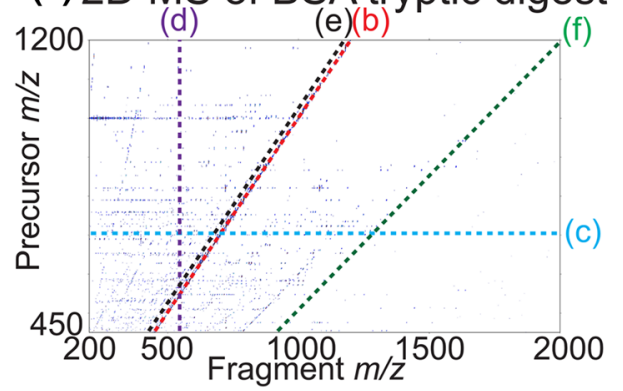

(b) Autocorrelation line

(c) Fragment ion scan of $\mathrm{m} / \mathrm{z} 710.3$

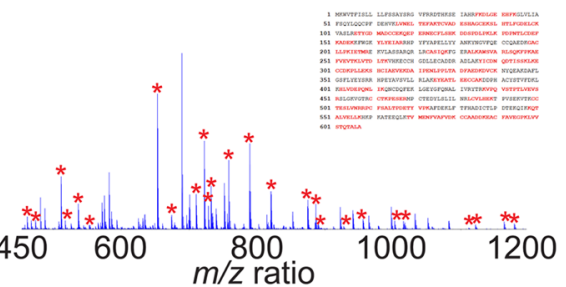

(e) Neutral loss line (18 Da/2)

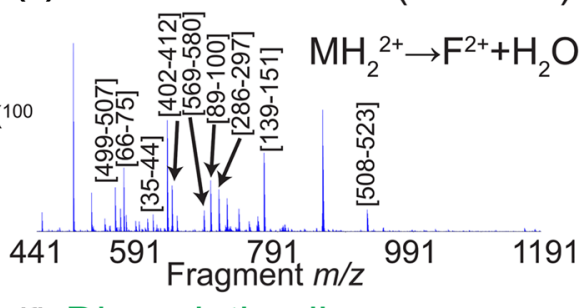

(f) Dissociation line

(d) Precursor ion scan of $\mathrm{m} / \mathrm{z} 549.27$

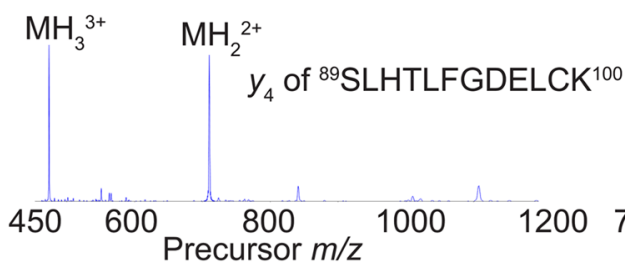

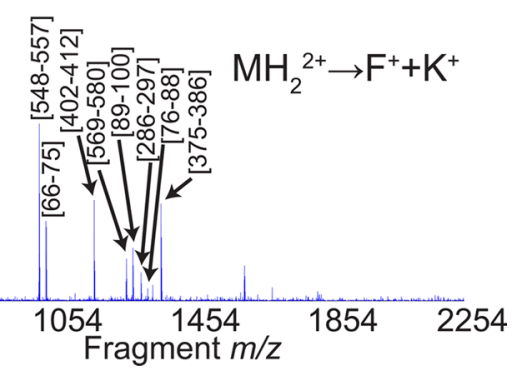


$p$ is the charge loss during the fragmentation, and $M$ is the mass loss during the fragmentation.

Equation 15 can be used for neutral losses as well (van Agthoven et al. 2015):

$(m / z)_{p}=(m / z)_{f}+\frac{M}{n}$

In the 2D mass spectrum, neutral loss lines are parallel to the autocorrelation line, and the distance between them measures the neutral loss mass and the charge of the precursors and fragments. Figure 4e shows the neutral loss line for loss of $18 \mathrm{Da}$ for doubly charged ions (i.e. water loss). Water loss in IRMPD can be indicative of a glutamate residue in a peptide, and all the peptide ions assigned on the neutral loss line contain a glutamate residue.

Figure $4 \mathrm{f}$ shows the dissociation line for loss of lysine by doubly charged precursor ions. Because lysine is a chargecarrying residue, loss of lysine happens together with charge loss. This dissociation line shows the peptides that have a lysine at the C-terminus. Because dissociation lines cross each other, a useful test to determine if a peak on a dissociation line actually corresponds to the dissociation that is sought is to check the charge state of its isotopic distribution against the charge state of the dissociation line.

Equation 15 can also be adapted for electron capture lines in ECD, in which $p$ electrons are captured and there is no mass change (the mass of an electron is considered negligible compared to the mass of the ions) (Floris et al. 2018c; van Agthoven et al. 2012):

$(m / z)_{p}=\frac{n-p}{n}(m / z)_{f}$

During fragmentation, isotopes from the precursor ion can end up in one fragment or its complementary fragment. For example, in the top-down analysis of ubiquitin in 2D IRMPD MS, the precursor ion $\mathrm{MH}_{7}^{7+}$ can dissociate into $y_{18}^{2+}$ and its complementary fragment $b_{59}^{5+}$. The elemental composition is $\mathrm{C}_{378} \mathrm{H}_{636} \mathrm{~N}_{105} \mathrm{O}_{118} \mathrm{~S}$ for $\mathrm{MH}_{7}{ }^{7+}, \mathrm{C}_{93} \mathrm{H}_{157} \mathrm{~N}_{29} \mathrm{O}_{26}$ for $y_{18}^{2+}$, and $\mathrm{C}_{285} \mathrm{H}_{475} \mathrm{~N}_{76} \mathrm{O}_{93} \mathrm{~S}$ for $b_{59}^{5+}$. Each ${ }^{13} \mathrm{C}$ isotope of $\mathrm{MH}_{7}^{7+}$ can be in $y_{18}^{2+}$ or $b_{59}^{5+}$ after fragmentation. The probability $p\left(y_{18}^{2+} / \mathrm{MH}_{7}^{7+}\right)$ for a precursor isotopologue of $\mathrm{MH}_{7}{ }^{7+}$ containing a number $n$ of ${ }^{13} \mathrm{C}$ isotopes (with probability $p\left(\mathrm{MH}_{7}^{7+}\right)$ of containing $n{ }^{13} \mathrm{C}$ isotopes) to dissociate into fragment isotopologue of $y_{18}^{2+}$ containing $p<n{ }^{13} \mathrm{C}$ isotopes (with probability $p\left(y_{18}^{2+}\right)$ corresponding to the probability of the fragment $y_{18}^{2+}$ containing the $p^{13} \mathrm{C}$ isotopes) and complementary fragment isotopologue of $b_{59}^{5+}$ (with probability $p\left(b_{59}^{5+}\right)$ corresponding to the probability of the fragment $b_{59}^{5+}$ containing the $n-p{ }^{13} \mathrm{C}$ isotopes) is:

$p\left(y_{18}^{2+} / \mathrm{MH}_{7}^{7+}\right)=\frac{p\left(y_{18}^{2+}\right) \times p\left(b_{59}^{5+}\right)}{p\left(\mathrm{MH}_{7}^{7+}\right)}$
Equation 18 yields the theoretical isotopic distribution of the $y_{18}^{2+}$ fragment in the 2D mass spectrum. Figure 5 shows the three-dimensional representation of isotopic distributions from the 2D IRMPD mass spectrum of ubiquitin. Figure $5 \mathrm{a}$ shows the isotopic distribution of $y_{18}^{2+}$ from $\mathrm{MH}_{7}{ }^{7+}$, and Fig. 5b shows the isotopic distribution of $\mathrm{MH}_{7}{ }^{7+}$ on the autocorrelation line. Figure $5 \mathrm{c}$ shows the theoretical isotopic distribution for ${ }^{13} \mathrm{C}$ isotopes for $y_{18}^{2+}$ from $\mathrm{MH}_{7}{ }^{7+}$, and Fig. $5 \mathrm{~d}$ the theoretical isotopic distribution for ${ }^{13} \mathrm{C}$ isotopes for $\mathrm{MH}_{7}{ }^{7+}$ on the autocorrelation line. Because the resolving power of the experimental 2D mass spectrum is limited along the precursor axis, the individual isotopic peaks cannot be separated in the experimental data, which explains the difference between the experimental and the theoretical distribution. As both the experimental and the theoretical distributions show, the spread of the isotopic distributions during a fragmentation is more complex than a simple dissociation line.

Neutral loss lines and dissociation lines can only be measured accurately along constant isotope lines (with the same number of ${ }^{13} \mathrm{C},{ }^{15} \mathrm{~N},{ }^{17} \mathrm{O},{ }^{18} \mathrm{O},{ }^{2} \mathrm{H}$, and ${ }^{34} \mathrm{~S}$ in both the precursor and the fragment ion). However, while mapping isotopic distributions in a 2D mass spectrum requires taking Eq. 18 into account for the fragmentation of large molecules (e.g. proteins), isotopic distributions of peptides with less than 15 residues and small molecules can be considered as restricted to the dissociation line (O'Connor et al. 1996; O'Connor and McLafferty 1995).

In any technique based on FT, any signal that is not perfectly sinusoidal generates harmonics at multiples of the ground frequency (or first harmonic) of the signal. In 2D MS, harmonics of the signal can occur in both the precursor and the fragment ion dimension. Figure 6 shows the 2D IRMPD spectrum of BSA from Fig. 4a in the frequency domain to show the harmonics in 2D MS.

In 2D mass spectra, harmonics are most visible as duplicates of the autocorrelation line as it is the location of the most intense signals. In frequency space, the equation of the autocorrelation line is:

$\omega_{\mathrm{v}}=\omega_{\mathrm{h}}-\omega_{\mathrm{off}}$

in which $\omega_{\mathrm{v}}$ is the modulation frequency of the precursor ion (plotted vertically), $\omega_{\mathrm{h}}$ is its cyclotron frequency (plotted horizontally), and the offset $\omega_{\text {off }}$ is due to the frequency shift induced by the continuous phase pulse generator, corresponding to the lowest excited frequency in $\mathrm{P}_{1}$ (van Agthoven et al. 2014). In Fig. 6a, the autocorrelation line is shown to have a frequency offset of $61.4 \mathrm{kHz}$, which corresponds to $\mathrm{m} / \mathrm{z}, 3000$ in the $12 \mathrm{~T}$ FT-ICR MS, which is the largest $m / z$ ratio excited in the pulse sequence.

In the fragment dimension, harmonics are generated because of the non-sinusoidal nature of the mirror current on the detection plates. The shape of the ICR cell is optimized 
(a) Experimental isotopic pattern of the $y_{18}{ }^{2+}$ fragment

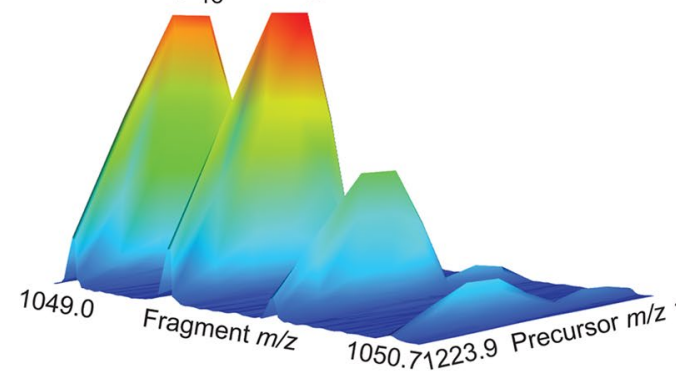

(c) Theoretical isotopic pattern

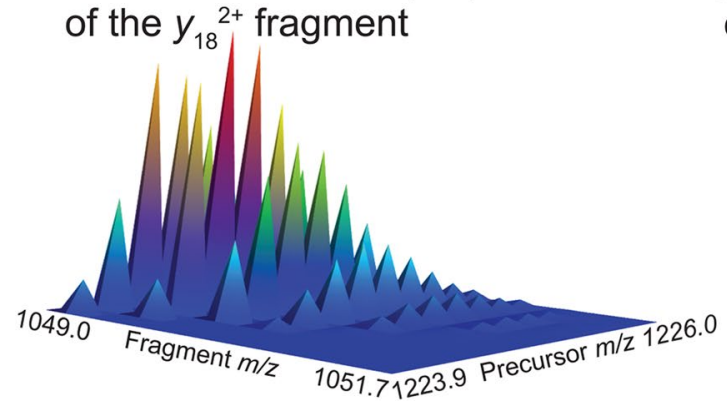

(b) Experimental isotopic pattern

of the $\mathrm{MH}_{7}^{7+}$ precursor

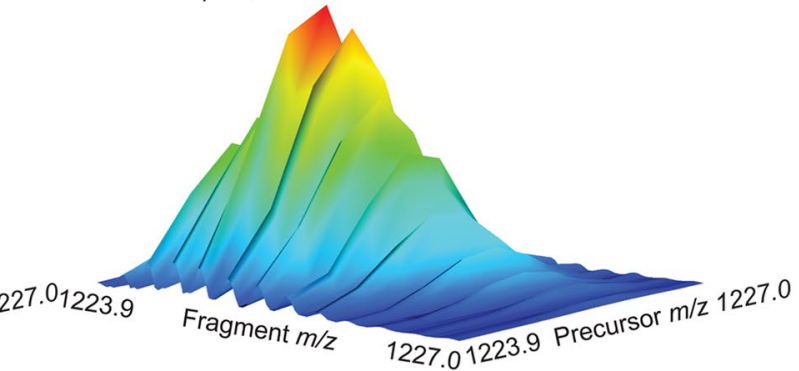

(d) Theoretical isotopic pattern of the $\mathrm{MH}_{7}^{7+}$ precursor

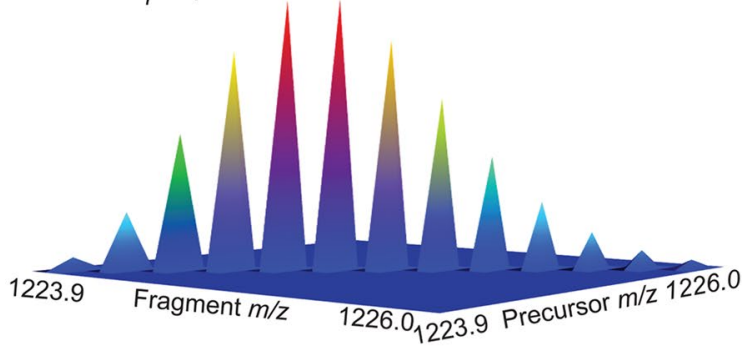

Fig. 5 Three-dimensional representation of isotopic patterns in the 2D IRMPD mass spectrum of ubiquitin. a The experimental isotopic pattern of the $y_{18}^{2+}$ fragment ion $(\mathrm{m} / \mathrm{z}, 1049.10$ for the monoisotopic peak) shows the that the contributions from the precursor isotopes in each peak in the isotopic pattern of the fragment ion cannot be separated due to the insufficient resolving power in the vertical dimension. b The experimental isotopic pattern of the $\mathrm{MH}_{7}{ }^{7+}$ precursor ion $(\mathrm{m} / z, 1223.809664$ for the monoisotopic peak) shows that the all the isotopic peaks of the precursor are on the autocorrelation line. $\mathbf{c}$ The theoretical isotopic pattern of the $y_{18}^{2+}$ fragment ion shows the contribution of each precursor isotope to the isotopic peaks of the fragment ion. $\mathbf{d}$ The theoretical isotopic pattern of the $\mathrm{MH}_{7}{ }^{7+}$ precursor ion shows the isotopic peaks of the precursor ion on the autocorrelation line

to limit the intensity of harmonics. In Fig. 6a, the horizontal harmonics of the autocorrelation line can be seen, with slopes of $1 / 2$ for the second harmonic and $1 / 3$ for the third harmonic, where the autocorrelation line itself is the first harmonic.

In the precursor dimension, harmonics of the autocorrelation line can be expressed as:

$\omega_{\mathrm{v}}=n\left(\omega_{\mathrm{h}}-\omega_{\text {off }}\right)$

in which $n$ is an integer. In Fig. 6a, two vertical harmonics of the autocorrelation line can be seen, with $n$ equalling 2 and 3 .

In addition to harmonics, 2D mass spectra also feature folded over signals. Because their frequency $\omega_{\mathrm{v}}$ is higher than the Nyquist frequency $\omega_{\mathrm{N}}$, these signals appear at $2 \omega_{\mathrm{N}}$ $-\omega_{\mathrm{v}}$ instead of $\omega_{\mathrm{v}}$. In frequency space, folded over harmonics of the autocorrelation line show up as lines with negative slopes (Marshall and Verdun 1990). Both the vertical harmonics discussed above fold over in the $2 \mathrm{D}$ spectrum.

In the conversion from frequency space to $\mathrm{m} / \mathrm{z}$ ratio space, the only straight line that is conserved as a straight line is the identity line (i.e. the first harmonic of the autocorrelation line). Digital demodulation reduces the number of harmonics visible in the 2D mass spectrum, which improves the ease of interpretation of the 2D mass spectra (van Agthoven et al. 2012). In the $m / z$ ratio domain, as shown in Fig. 6 b, harmonics of the autocorrelation line can be easily recognized because they are not straight lines as the $\mathrm{m} / \mathrm{z}$ ratios are a quasi-linear function of the inverse of frequencies in both dimensions. One side effect of digital demodulation is that they cause horizontal streaks at different multiples of $\omega_{\text {off }}$, which are the harmonics of the zero frequency now modulated by $\omega_{\text {off }}$. They can be seen in Fig. 6 at $61.4 \mathrm{kHz}$ and $122.8 \mathrm{kHz}$.

The effect of digital demodulation is to subtract $\omega_{\text {off }}$ from the frequencies of each signal. As stated above, the continuous wave frequency synthesizer used for pulse generation adds a constant frequency $\omega_{\text {off }}$ to all signals along the vertical $\omega_{1}$ axis. A signal at a frequency $\omega_{\mathrm{a}}+\omega_{\text {off }}$ before digital demodulation will therefore have a frequency at $\omega_{\mathrm{a}}$ after demodulation. Its second harmonic, which is at a frequency of $2\left(\omega_{\mathrm{a}}+\omega_{\text {off }}\right)$ before digital demodulation, will have a frequency of $2 \omega_{\mathrm{a}}+\omega_{\text {off }}$ after digital demodulation. While unavoidable, the presence of harmonics in a 2D mass spectrum can needlessly complicate data interpretation; harmonics have proven to be useful when using IR-ECD 
(a) 2D frequency spectrum of BSA tryptic digest with harmonics

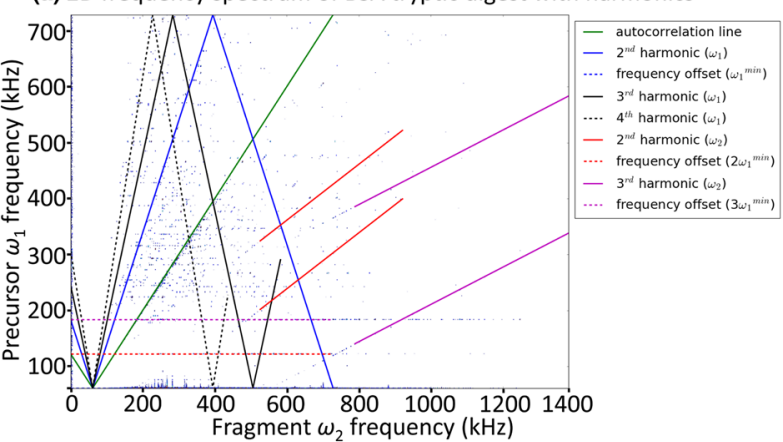

(b) 2D mass spectrum of BSA tryptic digest with harmonics



Fig. 6 a 2D IRMPD spectrum of bovine serum albumin tryptic digest in the frequency domain. The position of the harmonics of the autocorrelation line are shown in both $\omega_{1}$ and $\omega_{2}$ axes, the rebound of the lines on the top and bottom of the frequency spectrum corresponds to the aliasing of the signal at frequencies higher than the Nyquist frequency. b 2D IRMPD spectrum in the $\mathrm{m} / \mathrm{z}$ domain. The autocorrelation line is conserved but the other harmonics are curved, due to the inverse relationship between cyclotron frequencies and $\mathrm{m} / \mathrm{z}$ ratio (cf. Eq. 1)

as a fragmentation method, by enabling the differentiation of N-terminal and C-terminal fragments for peptides (van Agthoven et al. 2018).

2D mass spectra show vertical streaks along the peaks with the highest intensities. These streaks are called scintillation noise and are probably caused by fluctuations of the number of ions in the ICR cell (van der Rest and Marshall 2001). Figure 7a shows the 2D IRMPD mass spectrum of BSA shown in Fig. 4 with the scintillation noise. Scintillation noise is non-additive and roughly proportional to the signal $S(\mathrm{t})$ :

$S(t)=A(1+n(t)) \sqrt{1+\cos \omega t}$

in which $A$ is the amplitude of the signal, $\omega$ its frequency, and $n(t)$ the scintillation noise. Because of its proportionality to the signal, the impact of scintillation noise cannot be decreased by signal accumulation. Scintillation noise is

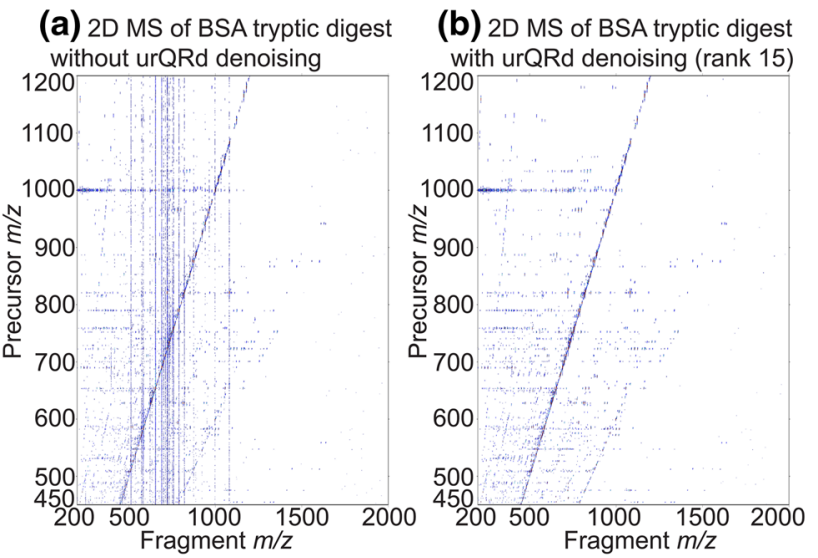

Fig. 7 a 2D IRMPD mass spectrum of bovine serum albumin without denoising. b 2D IRMPD mass spectrum of bovine serum albumin after urQRd denoising (rank 15). The urQRd denoising removes the vertical noise stripes

an important cause of misinterpretation of 2D mass spectra (van Agthoven et al. 2011a).

Denoising algorithms can be applied to vertical columns before FT in the vertical dimension. The first denoising algorithm used for processing 2D MS is an algorithm initially proposed by Cadzow et al. and relies on data predictability, i.e. the fact that for each transient, in the absence of noise, there is a number $N$ of data points, related to the number of signal present in the transient, after which it is possible to predict the $(N+1)^{\text {th }}$ data points (Cadzow and $\left.\mathrm{Wu} 1987\right)$ :

$S_{N+1}+\sum_{k=1}^{N} a_{k} S_{k}=0$

in which $a_{\mathrm{k}}$ are factors. An $n \times p$ sized Toeplitz matrix $T$ can therefore be composed from the data points:

$T=\left[\begin{array}{cccc}S_{n+1} & S_{n} & \cdots & S_{1} \\ S_{n+2} & S_{n+1} & \cdots & S_{2} \\ \vdots & \vdots & \vdots & \vdots \\ S_{n+p} & S_{n+p-1} & \cdots & S_{p}\end{array}\right]$

$T$ can be decomposed into eigenvalues and eigenvectors. If $n$ is higher than $N$, then some eigenvalues of $T$ will be zero. In the presence of noise, $T$ has no eigenvalues that are equal to zero. The Cadzow algorithm sets the smallest eigenvalues of $T$ to zero and reconstructs the denoised dataset from the denoised Toeplitz matrix.

The main advantage of the Cadzow algorithm is that no assumptions are made on the noise or signal power. The only parameter is the number of frequencies in the signal related to $N / 2$, since this number determines how many eigenvalues correspond to the signal as opposed to noise. Unfortunately, 
in 2D MS, the number of frequencies is not known a priori and varies from column to column. Furthermore, the algorithm is very time-consuming and implies an SVD computation, with a total processing time nearly proportional to $n^{3}$, in which $n$ is the number of data points (van Agthoven et al. 2011a).

An alternative algorithm, called uncoiled random $\mathrm{QR}$ denoising ( $\mathrm{rQRRd}$ ), relies on QR decomposition of a matrix randomly sampled from the data rather than an SVD decomposition. In the urQRd algorithm, the number of frequencies in the signal is not as crucial as in the Cadzow algorithm, making it a much more flexible than the Cadzow algorithm. The processing time for urQRd denoising is observed to be proportional to $n^{1.10}$, which corresponds to a gain of almost three orders of magnitude in real cases. The urQRd denoising algorithm can therefore be applied to datasets of almost unlimited size (Chiron et al. 2014). Figure 7b shows the 2D IRMPD mass spectrum of BSA from Fig. 4 after denoising with the urQRd algorithm. A side-by-side comparison with the raw spectrum shows that urQRd is effective in removing noise signals without removing the useful signal.

\section{Conclusion}

Two-dimensional mass spectrometry was first introduced in 1987, but it has only seen significant development since 2010 , because of the computational demands to store and process datasets and the impact of scintillation noise on the spectra. Since then, the development of 2D MS and the urQRd algorithm have enabled applications in the analysis of small molecules, proteomics (both top-down and bottomup), and polymers (Floris et al. 2016, 2017, 2018c; Simon et al. 2016; van Agthoven et al. 2015; van Agthoven et al. 2016). 2D MS has also been applied for $\mathrm{MS}^{3}$ experiments on proteins without ion isolation (Floris et al. 2018a, b). Because of the multiplexing of the data inherent to Fourier analysis, 2D MS has capabilities that other DDA and DIA techniques do not have in terms of correlation between precursor and fragment ions, especially for overlapping isotopic distributions (van Agthoven et al. 2016).

As an analytical technique, 2D MS is still in development. Alternative pulse sequences using stored waveform ion radius modulation (SWIM) enable the development of 2D MS in mass analysers other than the FT-ICR MS (Ross et al. 1993, 2002; van Agthoven and O'Connor 2017). Techniques that approach 2D MS are being developed for quadrupolar ion traps (Snyder et al. 2018). Non-uniform sampling acquisition has been shown to increase resolving power and shorten experiment durations (Bray et al. 2017). Theoretical studies enable a better understanding of the phenomena at play in a two-dimensional mass spectrometry experiment (Sehgal et al. 2016). Two-dimensional mass spectrometry has the potential to open up the entire field of mass spectrometry and to increase the structural information available on complex samples by several orders of magnitude.

Acknowledgements M.v.A. dedicates this article to the memory of Johannes W. Olsthoorn. The authors thank Ms. Alice Lynch, Dr. Lionel Chiron, Dr. Christopher Wootton, and Dr. Mark P. Barrow for their help in generating the artwork. M.v.A. and P.B.O. would like to thank the Centre for Scientific Computing at the University of Warwick for the use of their server for data processing. M.v.A. and P.B.O. would like to thank the Warwick Impact Fund Proof-of-Concept Award and the EPSRC Impact Acceleration Award, the Engineering and Physical Sciences Research Council for grant number EP/J000302/1 and grant number EP/N021630/1, the Biotechnology and Biological Sciences Research Council for grant number BBP021875/1, and the Royal Society Translation Award. C.R. and M.-A. D. thank the Agence Nationale de la Recherche (ANR, France; call, Défi de tous le savoirs 2014; Grant, ONE_SHOT_FT-ICR_MS_2D), the Défi CNRS MASTODONS (Grant, TABASCO), and the French FT-ICR MS network (FR CNRS 3624, Réseau National de Spectrometrie de Masse FT-ICR à très haut champ) for their financial support.

OpenAccess This article is distributed under the terms of the Creative Commons Attribution 4.0 International License (http://creativeco mmons.org/licenses/by/4.0/), which permits unrestricted use, distribution, and reproduction in any medium, provided you give appropriate credit to the original author(s) and the source, provide a link to the Creative Commons license, and indicate if changes were made.

\section{References}

Agarwal A, Diedrich JK, Julian RR (2011) Direct elucidation of disulfide bond partners using ultraviolet photodissociation mass spectrometry. Anal Chem 83:6455-6458. https://doi.org/10.1021/ ac201650v

Aizikov K, Mathur R, O'Connor PB (2009) The spontaneous loss of coherence catastrophe in fourier transform ion cyclotron resonance mass spectrometry. J Am Soc Mass Spectrom 20:247-256. https://doi.org/10.1016/j.jasms.2008.09.028

Amster IJ (1996) Fourier transform mass spectrometry. J Mass Spectrom 31:1325-1337. https://doi.org/10.1002/(SICI)10969888(199612)31:12\%3c1325:AID-JMS453\%3e3.0.CO;2-W

Bensimon M, Zhao G, Gäumann T (1989) A method to generate phase continuity in two-dimensional Fourier transform ion cyclotron resonance mass spectrometry. Chem Phys Lett 157:97-100. https ://doi.org/10.1016/0009-2614(89)87215-X

Biemann K (2002) Four decades of structure determination by mass spectrometry: from alkaloids to heparin. J Am Soc Mass Spectrom 13:1254-1272. https://doi.org/10.1016/s1044-0305(02)00441-5

Biemann K (2015) Structure determination of natural products by mass spectrometry. Ann Rev Anal Chem 8:1-19. https://doi. org/10.1146/annurev-anchem-071114-040110

Bodenhausen G, Kogler H, Ernst RR (1984) Selection of coherencetransfer pathways in NMR pulse experiments. J Magn Reson 58:370-388. https://doi.org/10.1016/0022-2364(84)90142-2

Boldin IA, Nikolaev EN (2009) Theory of peak coalescence in Fourier transform ion cyclotron resonance mass spectrometry. Rapid Commun Mass Spectrom 23:3213-3219. https://doi.org/10.1002/ rcm.4231

Bray F, Bouclon J, Chiron L, Witt M, Delsuc M-A, Rolando C (2017) Nonuniform sampling acquisition of two-dimensional fourier transform ion cyclotron resonance mass spectrometry for increased mass resolution of Tandem mass spectrometry precursor 
ions. Anal Chem 89:8589-8593. https://doi.org/10.1021/acs.analc hem. $7 \mathrm{~b} 01850$

Budnik BA, Haselmann KF, Zubarev RA (2001) Electron detachment dissociation of peptide di-anions: an electron-hole recombination phenomenon. Chem Phys Lett 342:299-302. https://doi. org/10.1016/s0009-2614(01)00501-2

Cadzow JA, Wu M-M (1987) Analysis of transient data in noise. In: IEE Proceedings Part F Communications, radar and signal processing 134:69-78 https://doi.org/10.1049/ip-f-1:19870013

Chapman JD, Goodlett DR, Masselon CD (2014) Multiplexed and dataindependent tandem mass spectrometry for global proteome profiling. Mass Spectrom Rev 33:452-470. https://doi.org/10.1002/ mas. 21400

Chiron L, van Agthoven MA, Kieffer B, Rolando C, Delsuc M-A (2014) Efficient denoising algorithms for large experimental datasets and their applications in Fourier transform ion cyclotron resonance mass spectrometry. Proc Natl Acad Sci USA 111:13851390. https://doi.org/10.1073/pnas.1306700111

Chiron L, Coutouly M-A, Starck J-P, Rolando C, Delsuc M-A (2016) SPIKE a processing software dedicated to Fourier spectroscopies arXivorg, e-Print Arch, Phys:1-13. arXiv:1608.06777

Cody RB, Freiser BS (1979) Electron impact excitation of ions from organics: an alternative to collision induced dissociation. Anal Chem 51:547-551. https://doi.org/10.1021/ac50040a022

Comisarow MB (1978) Signal modeling for ion cyclotron resonance. Journal of Chemical Physics 69:4097-4104. https://doi. org/10.1063/1.437143

Comisarow MB, Marshall AG (1974) Frequency-sweep Fourier transform ion cyclotron resonance spectroscopy. Chem Phys Lett 26:489-490. https://doi.org/10.1016/0009-2614(74)80397-0

Cramer CN, Brown JM, Tomczyk N, Nielsen PK, Haselmann KF (2016) Electron transfer dissociation of all ions at all times, MSETD, in a quadrupole time-of-flight (Q-ToF) mass spectrometer. J Am Soc Mass Spectrom. https://doi.org/10.1007/s13361-016-1538-2

de Hoffmann E, Stroobant V (2007) Mass spectrometry: principles and applications, 3rd edn. Wiley, Chichester

de Koning LJ, Nibbering NMM, van Orden SL, Laukien FH (1997) Mass selection of ions in a Fourier transform ion cyclotron resonance trap using correlated harmonic excitation fields (CHEF). Int J Mass Spectrom Ion Process 165(166):209-219. https://doi. org/10.1016/s0168-1176(97)00186-9

Decaestecker TN, Clauwaert KM, Van Bocxlaer JF, Lambert WE, Van den Eeckhout EG, Van Peteghem CH, De Leenheer AP (2000) Evaluation of automated single mass spectrometry to tandem mass spectrometry function switching for comprehensive drug profiling analysis using a quadrupole time-of-flight mass spectrometer. Rapid Commun Mass Spectrom 14:1787-1792. https:// doi.org/10.1002/1097-0231(20001015)14:19\%3c1787:aid-rcm94 $\% 3 \mathrm{e} 3.0 . \mathrm{co} ; 2$-s

Delsuc M-A (1988) Spectral representation of 2D NMR spectra by hypercomplex numbers. J Magn Reson 77:119-124. https://doi. org/10.1016/0022-2364(88)90036-4

Denisov E, Damoc E, Lange O, Makarov A (2012) Orbitrap mass spectrometry with resolving powers above $1,000,000$. Int J Mass Spectrom. 325:80-85. https://doi.org/10.1016/j.ijms.2012.06.009

Ernst RR, Bodenhausen G, Wokaun A (1987) Principles of nuclear magnetic resonance in one and two dimensions, 1st edn. Clarendon Press, Oxford

Floris F et al (2016) 2D FT-ICR MS of calmodulin: a top-down and bottom-Up approach. J Am Soc Mass Spectrom 27:1531-1538. https://doi.org/10.1007/s13361-016-1431-z

Floris F, Vallotto C, Chiron L, Lynch AM, Barrow MP, Delsuc M-A, O'Connor PB (2017) Polymer analysis in the second dimension: preliminary studies for the characterization of polymers with $2 \mathrm{D}$ MS. Anal Chem 89:9892-9899. https://doi.org/10.1021/acs.analc hem.7b02086
Floris F, Chiron L, Lynch AM, Barrow MP, Delsuc M-A, O’Connor PB (2018a) Application of tandem two-dimensional mass spectrometry for top-down deep sequencing of calmodulin. J Am Soc Mass Spectrom. https://doi.org/10.1007/s13361-018-1978-y

Floris F et al (2018b) Bottom-up two-dimensional electron-capture dissociation mass spectrometry of calmodulin. J Am Soc Mass Spectrom. https://doi.org/10.1007/s13361-017-1812-y

Floris F, Chiron L, Lynch AM, Barrow MP, Delsuc M-A, O'Connor PB (2018b) Top-down deep sequencing of ubiquitin using twodimensional mass spectrometry analytical chemistry (Washington, DC, United States) 90:7302-7309 https://doi.org/10.1021/acs. analchem. $8 \mathrm{~b} 00500$

Francl TJ, Sherman MG, Hunter RL, Locke MJ, Bowers WD, McIver RT Jr (1983) Experimental determination of the effects of space charge on ion cyclotron resonance frequencies. Int J Mass Spectrom Ion Processes 54:189-199. https://doi.org/10.1016/01681176(83)85017-4

Garnier N, Rolando C, Hotje JM, Tokarski C (2009) Analysis of archaeological triacylglycerols by high resolution nano ESI, FTICR MS and IRMPD MS/MS: application to 5th century BC-4th century AD oil lamps from Olbia (Ukraine). Int J Mass Spectrom Ion Process 284:47-56. https://doi.org/10.1016/j.jims.2009.03.003

Gerlich D (1992) Inhomogeneous RF fields: a versatile tool for the study of processes with slow ions. Adv Chem Phys 82:1-176. https://doi.org/10.1002/9780470141397.ch1

Gillet LC et al (2012) Targeted data extraction of the MS/MS spectra generated by data-independent acquisition: a new concept for consistent and accurate proteome analysis. Mol Cell Proteom 11:O111-016717. https://doi.org/10.1074/mcp.o111.016717

Gorshkov MV, Nikolaev EN (1993) Optimal cyclotron radius for high resolution FT-ICR spectrometry. Int J Mass Spectrom Ion Processes 125:1-8. https://doi.org/10.1016/0168-1176(93)80012-4

Guan S, Jones PR (1989) A theory for two-dimensional Fourier-transform ion cyclotron resonance mass spectrometry. J Chem Phys 91:5291-5295. https://doi.org/10.1063/1.457575

Guan S, McIver RT Jr (1990) Optimal phase modulation in stored wave form inverse Fourier transform excitation for Fourier transform mass spectrometry. I. Basic algorithm. J Chem Phys 92:58415846. https://doi.org/10.1063/1.458405

Hakansson K, Cooper HJ, Emmett MR, Costello CE, Marshall AG, Nilsson CL (2001) Electron capture dissociation and infrared multiphoton dissociation MS/MS of an N-glycosylated tryptic peptide to yield complementary sequence information. Anal Chem 73:4530-4536. https://doi.org/10.1021/ac0103470

Haselmann KF, Budnik BA, Kjeldsen F, Nielsen ML, Olsen JV, Zubarev RA (2002) Electronic excitation gives informative fragmentation of polypeptide cations and anions. Eur J Mass Spectrom 8:117-121. https://doi.org/10.1255/ejms.479

Jebanathirajah JA et al (2005) Characterization of a new qQq-FTICR mass spectrometer for post-translational modification analysis and top-down tandem mass spectrometry of whole proteins. J Am Soc Mass Spectrom 16:1985-1999. https://doi.org/10.1016/j.jasms .2005.08.008

Jerri AJ (1977) The Shannon sampling theorem; its various extensions and applications: a tutorial review. Proc IEEE 65:1565-1596. https://doi.org/10.1109/proc.1977.10771

Kaczorowska MA, Cooper HJ (2008) Electron capture dissociation, electron detachment dissociation, and collision-induced dissociation of polyamidoamine (PAMAM) dendrimer ions with amino, amidoethanol, and sodium carboxylate surface groups. J Am Soc Mass Spectrom 19:1312-1319. https://doi.org/10.1016/j.jasms 2008.06.016

Kruger NA, Zubarev RA, Horn DM, McLafferty FW (1999) Electron capture dissociation of multiply charged peptide cations. Int J Mass Spectrom 185(186/187):787-793. https://doi.org/10.1016/ s1387-3806(98)14215-x 
Kuo FF (1966) System analysis by digital computer, 1st edn. Wiley, New York

Ledford EB Jr, Rempel DL, Gross ML (1984) Space charge effects in Fourier transform mass spectrometry. II. Mass calibration. Anal Chem 56:2744-2748. https://doi.org/10.1021/ac00278a027

Little DP, Speir JP, Senko MW, O'Connor PB, McLafferty FW (1994) Infrared multiphoton dissociation of large multiply charged ions for biomolecule sequencing. Anal Chem 66:2809-2815

Madsen JA, Boutz DR, Brodbelt JS (2010) Ultrafast ultraviolet photodissociation at $193 \mathrm{~nm}$ and its applicability to proteomic workflows. J Proteome Res 9:4205-4214. https://doi.org/10.1021/pr100 $515 \mathrm{x}$

Marshall AG, Hendrickson CL (2008) High-resolution mass spectrometers. Ann Rev Anal Chem 1:579-599. https://doi.org/10.1146/ annurev.anchem.1.031207.112945

Marshall AG, Verdun FR (1990) Fourier transforms in NMR, optical and mass spectrometry. A user's handbook. Elsevier, Amsterdam. https://doi.org/10.1002/rcm.1290041027

Marshall AG, Wang TCL, Ricca TL (1984) Ion cyclotron resonance excitation/deexcitation: a basis for stochastic Fourier transform ion cyclotron mass spectrometry. Chem Phys Lett 105:233-236. https://doi.org/10.1016/0009-2614(84)85657-2

Marshall AG, Hendrickson CL, Jackson GS (1998) Fourier transform ion cyclotron resonance mass spectrometry: a primer. Mass Spectrom Rev 17:1-35. https://doi.org/10.1002/ (SICI)1098-2787(1998)17:1\%3c1:AID-MAS1\%3e3.0.CO;2-K

McDonald LA, Barbieri LR, Carter GT, Kruppa G, Feng X, Lotvin JA, Siegel MM (2003) FTMS structure elucidation of natural products: application to muraymycin antibiotics using ESI multiCHEF SORI-CID FTMSn, the top-down/bottom-up approach, and HPLC ESI capillary-skimmer CID FTMS. Anal Chem 75:27302739. https://doi.org/10.1021/ac0264731

Mosely JA, Smith MJP, Prakash AS, Sims M, Bristow AWT (2011) Electron-induced dissociation of singly charged organic cations as a tool for structural characterization of pharmaceutical type molecules. Anal Chem 83:4068-4075. https://doi.org/10.1021/ ac200045n

Nikolaev EN, Heeren RMA, Popov AM, Pozdneev AV, Chingin KS (2007) Realistic modeling of ion cloud motion in a fourier transform ion cyclotron resonance cell by use of a particle-in-cell approach. Rapid Commun Mass Spectrom 21:3527-3546

Nikolaev EN, Boldin IA, Jertz R, Baykut G (2011) Initial experimental characterization of a new ultra-high resolution FTICR cell with dynamic harmonization. J Am Soc Mass Spectrom 22:1125-1133. https://doi.org/10.1007/s13361-011-0125-9

Nikolaev EN, Jertz R, Grigoryev A, Baykut G (2012) Fine structure in isotopic peak distributions measured using a dynamically harmonized fourier transform ion cyclotron resonance cell at $7 \mathrm{~T}$. Anal Chem 84:2275-2283. https://doi.org/10.1021/ac202804f

O'Connor PB, McLafferty FW (1995) High-resolution ion isolation with the ion cyclotron resonance capacitively coupled open cell. J Am Soc Mass Spectrom 6:533-535. https://doi.org/10.1016/10440305(95)00232-3

O'Connor PB, Little DP, McLafferty FW (1996) Isotopic assignment in large-molecule mass spectra by fragmentation of a selected isotopic peak. Anal Chem 68:542-545. https://doi.org/10.1021/ ac950599z

O'Connor PB et al (2006) A new hybrid electrospray Fourier transform mass spectrometer: design and performance characteristics. Rapid Commun Mass Spectrom 20:259-266. https://doi.org/10.1002/ rem.2307

Panchaud A, Scherl A, Shaffer SA, von Haller PD, Kulasekara HD, Miller SI, Goodlett DR (2009) Precursor acquisition independent from ion count: how to dive deeper into the proteomics ocean. Anal Chem 81:6481-6488. https://doi.org/10.1021/ac900888s
Peng J, Elias JE, Thoreen CC, Licklider LJ, Gygi SP (2003) Evaluation of multidimensional chromatography coupled with tandem mass spectrometry (LC/LC-MS/MS) for large-scale protein analysis: the yeast proteome. J Proteome Res 2:43-50. https:// doi.org/10.1021/pr025556v

Pfändler P, Bodenhausen G, Rapin J, Houriet R, Gäumann T (1987) Two-dimensional Fourier transform ion cyclotron resonance mass spectrometry. Chem Phys Lett 138:195-200. https://doi. org/10.1016/0009-2614(87)80367-6

Pfändler P, Bodenhausen G, Rapin J, Walser ME, Gäumann T (1988) Broad-band two-dimensional Fourier transform ion cyclotron resonance. J Am Chem Soc 110:5625-5628. https://doi.org/10.1021/ ja00225a006

Plumb RS, Johnson KA, Rainville P, Smith BW, Wilson ID, CastroPerez JM, Nicholson JK (2006) UPLC/MSE; a new approach for generating molecular fragment information for biomarker structure elucidation. Rapid Commun Mass Spectrom 20:1989-1994. https://doi.org/10.1002/rcm.2550

Racaud A, Antoine R, Joly L, Mesplet N, Dugourd P, Lemoine J (2009) Wavelength-tunable ultraviolet photodissociation (UVPD) of heparin-derived disaccharides in a linear ion trap. J Am Soc Mass Spectrom 20:1645-1651. https://doi.org/10.1016/j.jasms .2009.04.022

Ramaswamy R, Siders P, Marcus RA (1981) A simple classical model of infrared multiphoton dissociation. J Chem Phys 74:4418-4425. https://doi.org/10.1063/1.441683

Ross CW III, Guan S, Grosshans PB, Ricca TL, Marshall AG (1993) Two-dimensional Fourier transform ion cyclotron resonance mass spectrometry/mass spectrometry with stored-waveform ion radius modulation. J Am Chem Soc 115:7854-7861. https://doi. org/10.1021/ja00070a035

Ross CW, Simonsick WJ Jr, Aaserud DJ (2002) Application of stored waveform ion modulation 2D-FTICR MS/MS to the analysis of complex mixtures. Anal Chem 74:4625-4633. https://doi. org/10.1021/ac0201833

Ruger CP et al (2017) Comprehensive chemical comparison of fuel composition and aerosol particles emitted from a ship diesel engine by gas chromatography atmospheric pressure chemical ionisation ultra-high resolution mass spectrometry with improved data processing routines. Eur J Mass Spectrom 23:28-39

Sehgal AA, Pelupessy P, Rolando C, Bodenhausen G (2016) Theory for spiralling ions for 2D FT-ICR and comparison with precessing magnetization vectors in 2D NMR. Phys Chem Chem Phys 18:9167-9175. https://doi.org/10.1039/c6cp00641h

Shi SDH, Drader JJ, Freitas MA, Hendrickson CL, Marshall AG (2000) Comparison and interconversion of the two most common frequency-to-mass calibration functions for fourier transform ion cyclotron resonance mass spectrometry. Int J Mass Spectrom 195(196):591-598. https://doi.org/10.1016/S1387-3806(99)00226 $-2$

Simon HJ et al (2016) Uncoiling collagen: a multidimensional mass spectrometry study. Analyst 141:157-165. https://doi.org/10.1039/ C5AN01757B

Sleno L, Volmer DA (2004) Ion activation methods for tandem mass spectrometry. J Mass Spectrom 39:1091-1112. https://doi. org/10.1002/jms.703

Snyder DT, Szalwinski LJ, Wells JM, Cooks RG (2018) Logical MS/ MS scans: a new set of operations for tandem mass spectrometry. Analyst 143:5438-5452. https://doi.org/10.1039/C8AN01661E

Song H, Hakansson K (2012) Electron detachment dissociation and negative ion infrared multiphoton dissociation of electrosprayed intact proteins. Anal Chem 5:871-876. https://doi.org/10.1021/ ac202909z

Syka JEP, Coon JJ, Schroeder MJ, Shabanowitz J, Hunt DF (2004) Peptide and protein sequence analysis by electron transfer dissociation 
mass spectrometry. Proc Natl Acad Sci USA 101:9528-9533. https://doi.org/10.1073/pnas.0402700101

Tsybin YO, Witt M, Baykut G, Kjeldsen F, Hakansson P (2003) Combined infrared multiphoton dissociation and electron capture dissociation with a hollow electron beam in Fourier transform ion cyclotron resonance mass spectrometry. Rapid Commun Mass Spectrom 17:1759-1768. https://doi.org/10.1002/rcm.1118

Valeja SG, Kaiser NK, Xian F, Hendrickson CL, Rouse JC, Marshall AG (2011) Unit mass baseline resolution for an intact $148 \mathrm{kDa}$ therapeutic monoclonal antibody by fourier transform ion cyclotron resonance mass spectrometry. Anal Chem 83:8391-8395. https://doi.org/10.1021/ac202429c

van Agthoven MA, O'Connor PB (2017) Two-dimensional mass spectrometry in a linear ion trap, an in silico model. Rapid Commun Mass Spectrom 31:674-684. https://doi.org/10.1002/rcm.7836

van Agthoven MA, Coutouly M-A, Rolando C, Delsuc M-A (2011a) Two-dimensional Fourier transform ion cyclotron resonance mass spectrometry: reduction of scintillation noise using Cadzow data processing. Rapid Commun Mass Spectrom 25:1609-1616. https ://doi.org/10.1002/rcm.5002

van Agthoven MA, Delsuc M-A, Rolando C (2011b) Two-dimensional FT-ICR/MS with IRMPD as fragmentation mode. Int J Mass Spectrom 306:196-203. https://doi.org/10.1016/j. ijms.2010.10.034

van Agthoven MA, Chiron L, Coutouly M-A, Delsuc M-A, Rolando C (2012) Two-dimensional ECD FT-ICR mass spectrometry of peptides and glycopeptides. Anal Chem 84:5589-5595. https:// doi.org/10.1021/ac3004874

van Agthoven MA, Delsuc M-A, Bodenhausen G, Rolando C (2013) Towards analytically useful two-dimensional Fourier transform ion cyclotron resonance mass spectrometry. Anal Bioanal Chem 405:51-61. https://doi.org/10.1007/s00216-012-6422-8

van Agthoven MA, Chiron L, Coutouly M-A, Sehgal AA, Pelupessy P, Delsuc M-A, Rolando C (2014) Optimization of the discrete pulse sequence for two-dimensional FT-ICR mass spectrometry using infrared multiphoton dissociation. Int J Mass Spectrom 370:114-124. https://doi.org/10.1016/j.jims.2014.06.019

van Agthoven MA et al (2015) Differentiating fragmentation pathways of cholesterol by two-dimensional Fourier transform ion cyclotron resonance mass spectrometry. J Am Soc Mass Spectrom 26:21052114. https://doi.org/10.1007/s13361-015-1226-7

van Agthoven MA et al (2016) Two-dimensional mass spectrometry for proteomics, a comparative study with cytochrome c. Anal Chem 88:4409-4417. https://doi.org/10.1021/acs.analchem.5b04878 van Agthoven MA et al (2018) Can Two-dimensional IR-ecd mass spectrometry improve peptide de novo sequencing? Anal Chem 90:3496-3504. https://doi.org/10.1021/acs.analchem.7b05324

van der Rest G, Marshall AG (2001) Noise analysis for 2D tandem Fourier transform ion cyclotron resonance mass spectrometry. Int J Mass Spectrom 210:101-111

Vartanian VH, Anderson JS, Laude DA (1995) Advances in trapped ion cells for Fourier transform ion cyclotron resonance mass spectrometry. Mass Spectrom Rev 14:1-19. https://doi.org/10.1002/ mas. 1280140102

Wang TCL, Ricca TL, Marshall AG (1986) Extension of dynamic range in Fourier transform ion cyclotron resonance mass spectrometry via stored waveform inverse Fourier transform excitation. Anal Chem 58:2935-2938. https://doi.org/10.1021/ac00127a009

Wei J et al. (2016) Amyloid hydrogen bonding polymorphism evaluated by $15 \mathrm{~N}\{17 \mathrm{O}\}$ REAPDOR Solid-State NMR and ultra-high resolution fourier transform ion cyclotron resonance Mass Spectrometry Biochemistry 55:2065-2068 https://doi.org/10.1021/acs. biochem.5b01095

Wei J, Li H, Barrow MP, O'Connor PB (2013) Structural Characterization of chlorophyll-a by high resolution tandem mass spectrometry. J Am Soc Mass Spectrom 24:753-760. https://doi. org/10.1007/s13361-013-0577-1

Wills RH, O'Connor PB (2014) Structural characterization of actinomycin D using multiple ion isolation and electron induced dissociation. J Am Soc Mass Spectrom 25:186-195. https://doi. org/10.1007/s13361-013-0774-y

Wootton CA, Lam YPY, Willetts M, van Agthoven MA, Barrow MP, Sadler PJ, O'Connor PB (2017) Automatic assignment of metalcontaining peptides in proteomic LC-MS and MS/MS data sets. Analyst 142:2029-2037. https://doi.org/10.1039/c7an00075h

Zhang L-K, Rempel D, Pramanik BN, Gross ML (2005) Accurate mass measurements by Fourier transform mass spectrometry. Mass Spectrom Rev 24:286-309. https://doi.org/10.1002/mas.20013

Zubarev RA, Kelleher NL, McLafferty FW (1998) Electron capture dissociation of multiply charged protein cations. A nonergodic process. J Am Chem Soc 120:3265-3266. https://doi.org/10.1021/ ja973478k

Publisher's Note Springer Nature remains neutral with regard to jurisdictional claims in published maps and institutional affiliations. 